\title{
The Genetic Makeup and Expression of the Glycolytic and Fermentative Pathways Are Highly Conserved Within the Saccharomyces Genus
}

\author{
Francine J. Boonekamp ${ }^{1}$, Sofia Dashko ${ }^{1}$, Marcel van den Broek ${ }^{1}$, Thies Gehrmann², \\ Jean-Marc Daran ${ }^{1}$ and Pascale Daran-Lapujade ${ }^{1 *}$
}

${ }^{1}$ Department of Biotechnology, Delft University of Technology, Delft, Netherlands, ${ }^{2}$ Westerdijk Institute, Utrecht, Netherlands

\section{OPEN ACCESS}

Edited by:

Ed Louis,

University of Leicester

United Kingdom

Reviewed by:

Catherine Tesnière,

Institut National de la Recherche Agronomique Centre Montpellier,

France

Jing Hua Zhao,

University of Cambridge,

United Kingdom

*Correspondence:

Pascale Daran-Lapujade

p.a.s.daran-lapujade@tudelft.n

Specialty section:

This article was submitted to

Evolutionary and Genomic

Microbiology,

a section of the journal

Frontiers in Genetics

Received: 27 July 2018 Accepted: 08 October 2018 Published: 16 November 2018

Citation:

Boonekamp FJ, Dashko S, van den Broek M, Gehrmann T, Daran J-M and Daran-Lapujade P

(2018) The Genetic Makeup and Expression of the Glycolytic and Fermentative Pathways Are

Highly Conserved Within

the Saccharomyces Genus.

Front. Genet. 9:504.

doi: 10.3389/fgene.2018.00504
The ability of the yeast Saccharomyces cerevisiae to convert glucose, even in the presence of oxygen, via glycolysis and the fermentative pathway to ethanol has played an important role in its domestication. Despite the extensive knowledge on these pathways in $S$. cerevisiae, relatively little is known about their genetic makeup in other industrially relevant Saccharomyces yeast species. In this study we explore the diversity of the glycolytic and fermentative pathways within the Saccharomyces genus using S. cerevisiae, Saccharomyces kudriavzevii, and Saccharomyces eubayanus as paradigms. Sequencing data revealed a highly conserved genetic makeup of the glycolytic and fermentative pathways in the three species in terms of number of paralogous genes. Although promoter regions were less conserved between the three species as compared to coding sequences, binding sites for Rap1, Gcr1 and Abf1, main transcriptional regulators of glycolytic and fermentative genes, were highly conserved. Transcriptome profiling of these three strains grown in aerobic batch cultivation in chemically defined medium with glucose as carbon source, revealed a remarkably similar expression of the glycolytic and fermentative genes across species, and the conserved classification of genes into major and minor paralogs. Furthermore, transplantation of the promoters of major paralogs of $S$. kudriavzevii and $S$. eubayanus into S. cerevisiae demonstrated not only the transferability of these promoters, but also the similarity of their strength and response to various environmental stimuli. The relatively low homology of $S$. kudriavzevii and S. eubayanus promoters to their S. cerevisiae relatives makes them very attractive alternatives for strain construction in S. cerevisiae, thereby expanding the $S$. cerevisiae molecular toolbox.

Keywords: glycolysis, promoter characterization, Saccharomyces cerevisiae, Saccharomyces kudriavzevii, Saccharomyces eubayanus, transcription factor binding sites

\section{INTRODUCTION}

The yeast Saccharomyces cerevisiae is known for its fast fermentative metabolism, which has played an important role in its domestication (Sicard and Legras, 2011). S. cerevisiae converts glucose to ethanol via the Embden-Meyerhof-Parnas pathway of glycolysis and the fermentative pathway, encompassing a total of 12 enzymatic steps (Barnett, 2003; Barnett and Entian, 2005). While S. cerevisiae can respire glucose, leading to an ATP yield of 16 moles of ATP per mole of glucose, it favors alcoholic fermentation. Indeed, even in the presence of oxygen, glucose excess triggers 
ethanol formation in S. cerevisiae and its relatives from the Saccharomyces genus, a phenomenon known as the Crabtree effect (De Deken, 1966; Merico et al., 2007). To sustain the energy demand for growth and maintenance despite the low ATP yield of alcoholic fermentation ( 2 moles of ATP per glucose molecule), the glycolytic flux in $S$. cerevisiae can easily reach fluxes of 20-25 mmoles ethanol per gram dry weight per hour (SolisEscalante et al., 2015). This high activity of the glycolytic pathway is reflected in the remarkably high concentration of glycolytic enzymes in the cell, which can represent up to $30 \%$ of the total amount of soluble protein (Fraenkel, 2003; Carroll et al., 2011).

The genome of $S$. cerevisiae is characterized by a high genetic redundancy which can largely be attributed to a whole genome duplication event (Ohno, 1970; Wolfe and Shields, 1997). This redundancy is even more prominent among 'metabolic' genes and is remarkably elevated in the glycolytic and fermentative pathways of S. cerevisiae (Kellis et al., 2004; Kuepfer et al., 2005; Conant and Wolfe, 2007). These two pathways have been thoroughly investigated (and even established) in S. cerevisiae (Barnett, 2003; Van Heerden et al., 2015). With the exception of three steps that are catalyzed by single enzymes, i.e., phosphoglucose isomerase (Pgil), fructose-bisphosphate aldolase (Fba1), and triosephosphate isomerase (Tpi1), the glycolytic, and fermentative steps are catalyzed by at least two and potentially up to seven isoenzymes for alcohol dehydrogenase (Adh). However, not all isoenzymes are equally important for the glycolytic and fermentative activity. With the notable exception of Pfk1 and Pfk2, two isoenzymes forming a heterooctamer that are equally important for the functionality of phosphofructokinase (Heinisch et al., 1991; Arvanitidis and Heinisch, 1994), for each step, a single isoenzyme is responsible for the bulk of the glycolytic and fermentative flux. These so-called major isoenzymes are encoded by major paralogs, which expression is strong and constitutive (i.e., HXK2, TDH3, GPM1, ENO2, PYK1, PDC1, ADH1) (Solis-Escalante et al., 2015). Because of these properties, glycolytic promoters are often used to drive gene expression in engineered strains (Peng et al., 2015). Conversely the expression of minor paralogs is, in most instances, far lower than the expression of the corresponding major paralogs and is conditiondependent (Ciriacy, 1979; Boer et al., 2003; Knijnenburg et al., 2009; Solis-Escalante et al., 2015). Following duplication events, redundant genes can have different fates. If their presence brings additional benefits to the cell, either in their native form or via neo-functionalization, the gene and its duplicate will be retained in the genome, otherwise the redundant copy will be lost (Kellis et al., 2004; Conant and Wolfe, 2008). The fact that the glycolytic and fermentative pathways still contain many paralogs that do not display obvious new functions suggests that they might increase fitness under specific conditions. For example, PDC6 encoding a pyruvate decarboxylase with low sulfur amino acid content is specifically induced in sulfur limiting conditions (Fauchon et al., 2002; Boer et al., 2003). However, challenging this theory, it was recently shown that the simultaneous removal of all minor paralogs from the glycolytic and fermentative pathways had no detectable effect on S. cerevisiae physiology under a wide variety of conditions (Solis-Escalante et al., 2015).
The Saccharomyces genus consists of at least eight naturally occurring species which all evolved toward optimal performance in their niche, leading to different physiological characteristics (Replansky et al., 2008; Hittinger, 2013; Naseeb et al., 2017). For instance, Saccharomyces kudriavzevii, Saccharomyces uvarum and Saccharomyces eubayanus are cold-tolerant, and perform better than $S$. cerevisiae at temperatures below $20^{\circ} \mathrm{C}$ (ArroyoLópez et al., 2010; Masneuf-Pomarède et al., 2010; Salvadó et al., 2011; Hebly et al., 2015). Strains belonging to different Saccharomyces species can mate and form viable hybrids, some of which play an important role in the beverage industry. For instance Saccharomyces pastorianus, a hybrid of S. cerevisiae and S. eubayanus, is the main lager-brewing yeast (Bond, 2009) and hybrids of $S$. cerevisiae and $S$. kudriavzevii and of $S$. uvarum and S. eubayanus (known as S. bayanus) play an important role in beer and wine fermentation (González et al., 2006; González et al., 2008; Peris et al., 2012; Nguyen and Boekhout, 2017). The cold-tolerance of S. pastorianus and S. eubayanus has indubitably promoted the selection of their hybrids with $S$. cerevisiae in cold environments (Belloch et al., 2008; Arroyo-López et al., 2010; Libkind et al., 2011).

In a recent study, using a unique yeast platform enabling the swapping of entire essential pathways, it was shown that S. kudriavzevii glycolytic and fermentative pathways could be transplanted in S. cerevisiae and could efficiently complement the native pathways. Expression of the full set of S. kudriavzevii orthologs in $S$. cerevisiae, expressed from $S$. kudriavzevii promoters, resulted in enzyme activities and physiological responses remarkably similar to the parental strain carrying a full set of native $S$. cerevisiae genes. However, the impact of $S$. kudriavzevii promoters on transcriptional activity in S. cerevisiae was not explored (Kuijpers et al., 2016). Despite S. eubayanus and S. kudriavzevii industrial importance and the availability of their full genome sequence, remarkably little is known about the genetic makeup and transcriptional regulation of the glycolytic and fermentative pathways.

To address this knowledge gap, the present study explores the diversity of the glycolytic and fermentative pathways within the genus Saccharomyces, using the industrially relevant yeasts S. cerevisiae, S. eubayanus and S. kudriavzevii as paradigms. More precisely, the presence and sequence similarity between paralogs in these three yeasts were explored. Cultivation in bioreactors combined with transcriptome analysis was used to evaluate the presence of dominant paralogs in S. eubayanus and S. kudriavzevii and to compare the expression levels of glycolytic and fermentative orthologs in their native context. Finally, we explored transferability of $S$. kudriavzevii and S. eubayanus promoters by monitoring their expression and context-dependency upon transplantation in S. cerevisiae.

\section{MATERIALS AND METHODS}

\section{Strains and Culture Conditions}

All yeast strains used in this study are derived from the CEN.PK background (Entian and Kötter, 2007) and are listed in Table 1. Yeast cultures for transformation and genomic DNA isolation 
TABLE 1 | Strains table.

\begin{tabular}{ll}
\hline Strain & \multicolumn{1}{c}{ Genotype } \\
\hline Saccharomyces cerevisiae \\
CEN.PK113-5D & MATa URa3-52 HIS3 LEU2 TRP1 MAL2-8C SUC2 \\
CEN.PK113-7D & MATa URA3 HIS3 LEU2 TRP1 MAL2-8C SUC2
\end{tabular}

CEN.PK113-7D MATa URA3 HIS3 LEU2 TRP1 MAL2-8C SUC2
Plasmid for integration Source

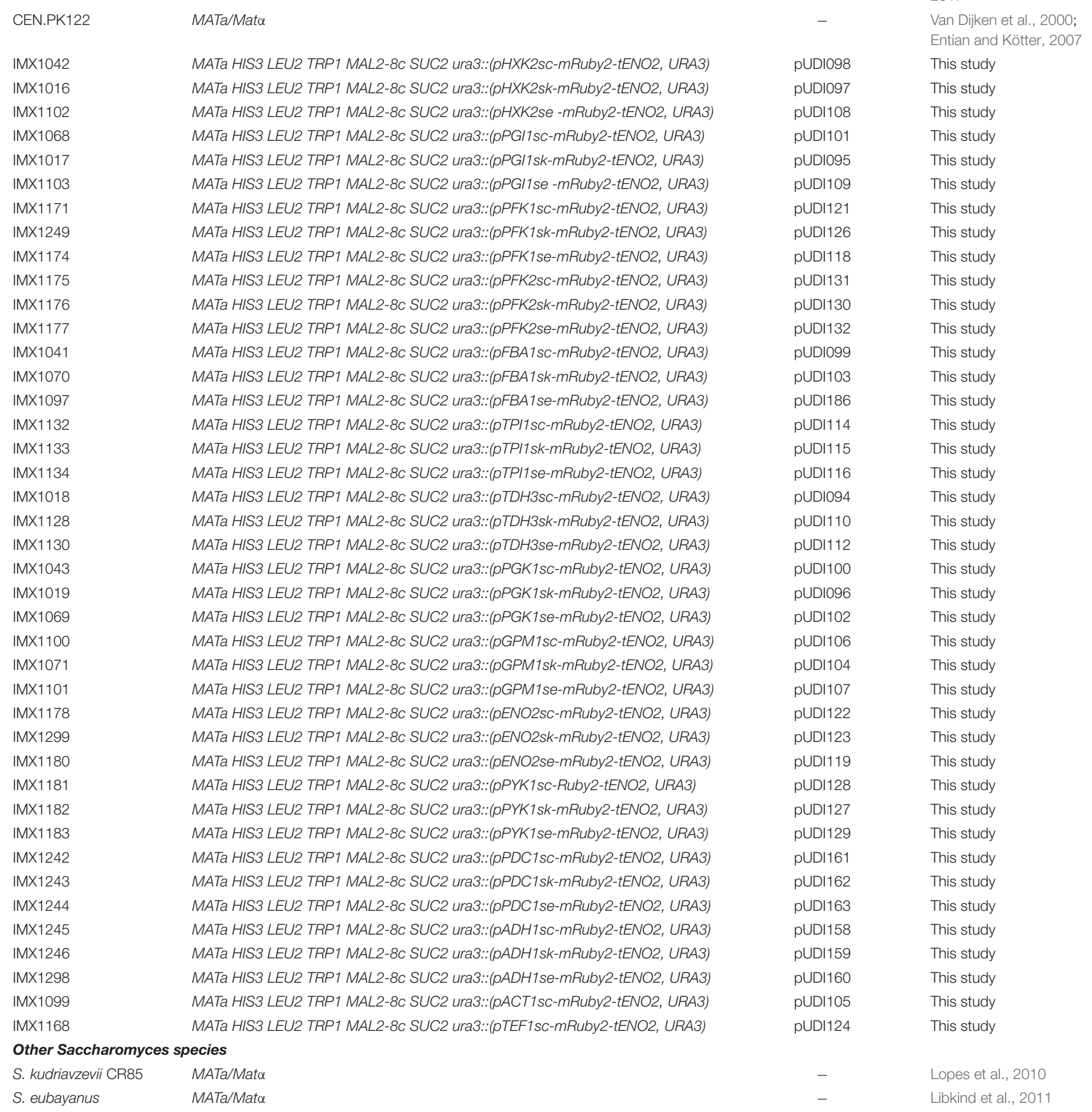

Entian and Kötter, 2007

Van Dijken et al., 2000; Entian and Kötter, 2007; Nijkamp et al., 2012; Salazar et al.,

Van Dijken et al., 2000; This study

This study

This study

This study

This study

This study

This study 
were grown in $500 \mathrm{~mL}$ shake flasks with $100 \mathrm{~mL}$ of complex, nonselective medium (YPD) containing $10 \mathrm{~g} \mathrm{~L}^{-1}$ Bacto Yeast extract, $20 \mathrm{~g} \mathrm{~L}^{-1}$ Bacto Peptone and $20 \mathrm{~g} \mathrm{~L}^{-1}$ glucose. Promoter regions were obtained from the strains S. cerevisiae CEN.PK113-7D (Van Dijken et al., 2000; Entian and Kötter, 2007; Nijkamp et al., 2012), S. kudriavzevii CR85 a wild isolate from oak bark (supplied by Prof. Querol and dr. Barrio, Universitat de València, Spain) (Lopes et al., 2010) and S. eubayanus CBS12357 (Libkind et al., 2011). The same strains were used for transcriptome analysis, with the exception of $S$. cerevisiae for which the diploid strain CEN.PK122 was used instead of the haploid CEN.PK113-7D (Entian and Kötter, 2007). All S. cerevisiae strains were grown at $30^{\circ} \mathrm{C}$ and S. kudriavzevii and S. eubayanus at $20^{\circ} \mathrm{C}$ in shake flasks at $200 \mathrm{rpm}$, unless different conditions are mentioned.

All transformations were done in S. cerevisiae CEN.PK1135D using the auxotrophic marker URA3 for selection. Synthetic medium containing $3 \mathrm{~g} \mathrm{~L}^{-1} \mathrm{KH}_{2} \mathrm{PO}_{4}, 0.5 \mathrm{~g} \mathrm{~L}^{-1} \mathrm{MgSO}_{4} \cdot 7 \mathrm{H}_{2} \mathrm{O}$, $5 \mathrm{~g} \mathrm{~L}^{-1}\left(\mathrm{NH}_{4}\right)_{2} \mathrm{SO}_{4}, 1 \mathrm{~mL} \mathrm{~L}{ }^{-1}$ of a trace element solution, and $1 \mathrm{~mL} \mathrm{~L}^{-1}$ of a vitamin solution was used (Verduyn et al., 1992). Synthetic medium supplemented with $20 \mathrm{~g} \mathrm{~L}^{-1}$ glucose (SMG) or $2 \%$ (vol/vol) ethanol (SMEtOH) was used for culture propagation where specified. For solid media $20 \mathrm{~g} \mathrm{~L}^{-1}$ agar was added prior to heat sterilization. For storage and propagation of plasmids Escherichia coli XL1-Blue (Agilent Technologies, Santa Clara, CA, United States) was used, and grown in lysogeny broth (LB) supplemented with ampicillin $\left(100 \mathrm{mg} \mathrm{L}^{-1}\right)$ (Bertani, 1951; Bertani, 2004). For the storage of yeast and E. coli strains $30 \%$ or $15 \%(\mathrm{v} / \mathrm{v})$ glycerol was added to exponentially growing cultures respectively, and aliquots were stored at $-80^{\circ} \mathrm{C}$.

\section{Molecular Biology Techniques}

For high fidelity PCR amplification Phusion high fidelity polymerase (Thermo Scientific, Landsmeer, Netherlands) was used according to manufacturer's instructions. To improve efficiency of the PCR reactions, primer concentrations were decreased from 500 to $200 \mathrm{nM}$ and the polymerase concentration was increased from 0.02 to $0.03 \mu \mathrm{L}^{-1}$. PCR products were treated with $1 \mu \mathrm{L}$ DpnI FastDigest restriction enzyme (Thermo Fisher Scientific) for $1 \mathrm{~h}$ at $37^{\circ} \mathrm{C}$ to remove residual circular templates. Afterward, the mixture was purified using GenElute ${ }^{\mathrm{TM}}$ PCR Clean-Up Kit (Sigma-Aldrich, St. Louis, MO) according to manufacturer's protocol. PCR for diagnostic purposes was done using DreamTaq PCR mastermix (Thermo Fisher Scientific) according to manufacturer's recommendations. Primers used in this study are listed in Supplementary Tables S1, S2. PCR products were resolved on $1 \%$ agarose gel with Trisacetate-EDTA (TAE) buffer. Genomic DNA used as template for PCR amplification of the promoter regions was isolated using YeaStar genomic DNA kit (Zymo Research, Orange, CA) according to manufacturer's protocol. Plasmids were extracted from E. coli using the GenElute plasmid miniprep kit (SigmaAldrich) according to manufacturer's description and eluted with miliQ water. Restriction analysis of plasmids was done using FastDigest restriction enzymes with FastDigest Green Buffer (Thermo Fisher Scientific) incubating for $30 \mathrm{~min}$ at $37^{\circ} \mathrm{C}$ according to manufacturer's recommendations.

\section{Promoters, Plasmids and Yeast Strain Construction}

A schematic overview of the subsequent plasmid and strain construction steps is provided in Figure 1. Plasmids used in this study are reported in Supplementary Table S3. The HXK2, PGI1, PFK1, PFK2, FBA1, TPI1, TDH3, PGK1, GPM1, ENO2, PYK1, PDC1 and ADH1, and reference TEF1 and ACT1 promoter regions of approximately $800 \mathrm{bp}$ (see Supplementary Table S4 for exact lengths) were PCR-amplified from S. cerevisiae CEN.PK113-7D, S. kudriavzevii CR85 and S. eubayanus CBS 12357 genomic DNA using primers listed in Supplementary Table S1. For compatibility with Golden Gate cloning, promoter sequences were flanked with $\mathrm{BsaI}$ and $\mathrm{BsmBI}$ restriction sites introduced as primer overhangs in the PCR amplification step.

The plasmid backbone was constructed by Golden Gate assembly using the collection of part plasmids provided in the Yeast Toolkit (Lee et al., 2015). To increase the efficiency of plasmid assembly, first a GFP dropout plasmid pUD428 was constructed containing a URA3 marker, AmpR selection marker, bacterial origin of replication, two connector fragments and a GFP gene surrounded by URA 3 upstream and downstream homology flanks (Supplementary Table S3). The correct assembly of plasmids was checked by restriction analysis. The GFP dropout cassette from pUD428 was subsequently replaced by the $m R u b y 2$ gene flanked by a promoter of interest and by the ENO2 terminator using Golden Gate cloning with BsaI. The reaction mixture was prepared with $1 \mu \mathrm{L}$ T4 DNA ligase buffer (Thermo Fisher Scientific), $0.5 \mu \mathrm{L}$ T7 DNA ligase (NEB New England Biolabs, Ipswich, MA), $0.5 \mu \mathrm{L}$ FastDigest Eco31I (BsaI) (Thermo Fisher Scientific) and $10 \mathrm{ng}$ of each DNA fragment. MiliQ $\mathrm{H}_{2} \mathrm{O}$ was added to a final volume of $10 \mu \mathrm{L}$. The assembly was done in a thermocycler using 25 cycles of restriction and ligation: $42^{\circ} \mathrm{C}$ for $2 \mathrm{~min}, 16^{\circ} \mathrm{C}$ for $5 \mathrm{~min}$, followed by a final digestion step $\left(60^{\circ} \mathrm{C}\right.$ for $\left.10 \mathrm{~min}\right)$ and an inactivation step $\left(80^{\circ} \mathrm{C}\right.$ for $\left.10 \mathrm{~min}\right)$. If one of the fragments contained an internal BsaI site, the final digestion and inactivation steps were omitted. $1 \mu \mathrm{L}$ of the assembly mix was transformed to E. coli (XL1-Blue) according to manufacturer's description and plated on selective LB medium. Correct ligation of the promoter-mRuby2-terminator construct in this plasmid resulted in the loss of the GFP gene, which could be easily screened based on colony color. Additional plasmid confirmation was done by restriction analysis.

Prior to transformation into yeast, the constructed plasmids containing the promoter of interest, the mRuby2 gene and the ENO2 terminator were linearized by digestion with NotI (FastDigest, Thermo Fisher Scientific) according to manufacturer's protocol for $30 \mathrm{~min}$ at $37^{\circ} \mathrm{C}$. $400 \mathrm{ng}$ of each plasmid was digested and the mixture was directly transformed to the strain CEN.PK113-5D in which the linearized plasmid was integrated in the ura3-52 locus. Yeast transformations were done according to Gietz and Woods (Gietz and Woods, 2002). Colonies were screened by PCR (Supplementary Table S2).

\section{Batch Cultivation in Bioreactors}

Samples for transcriptome analysis of S. cerevisiae (CEN.PK122), S. kudriavzevii (CR85) and S. eubayanus (CBS 12357) were obtained from aerobic batch cultures in bioreactors performed in 


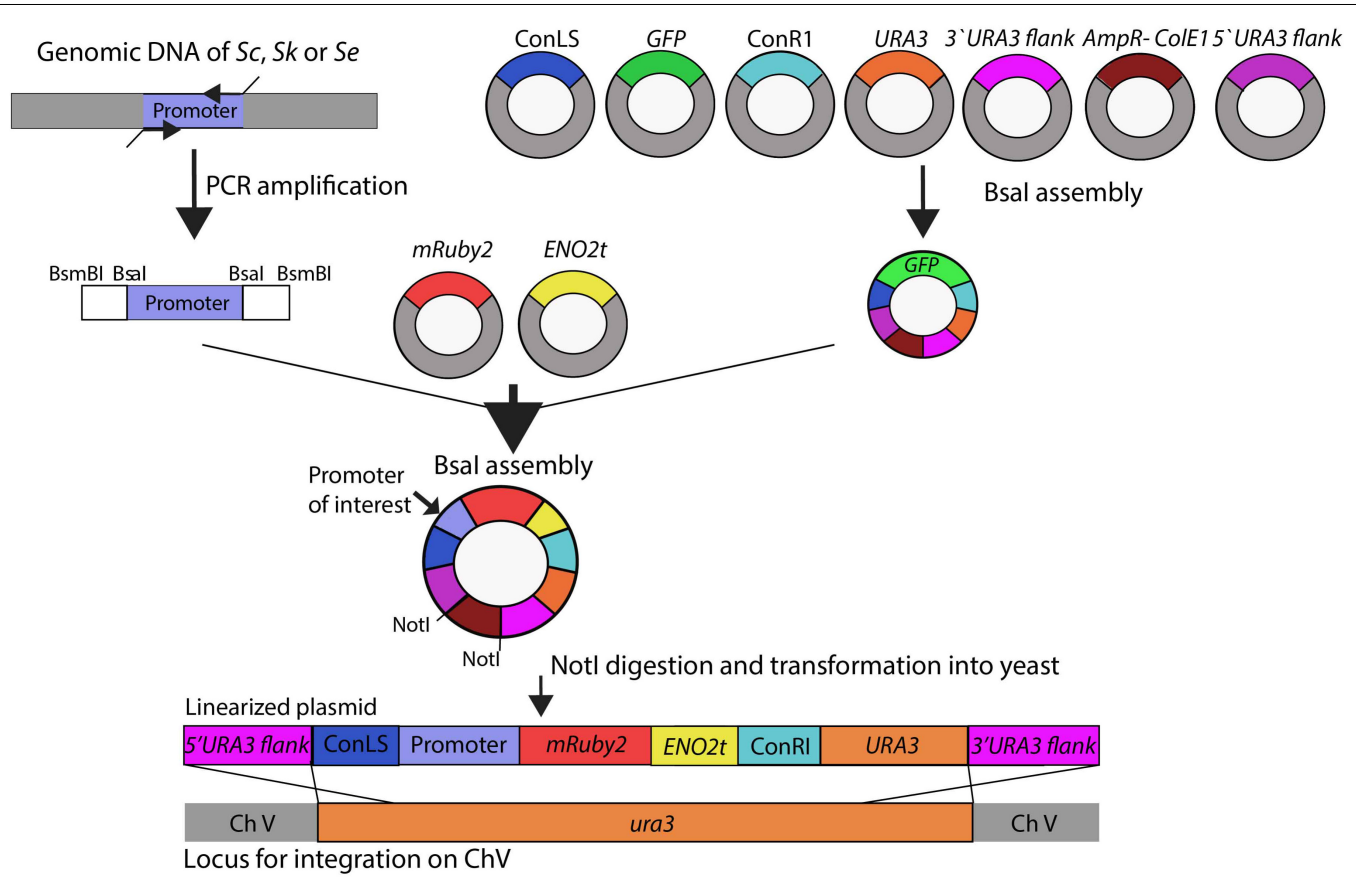

FIGURE 1 | Schematic representation of the strain construction workflow. Glycolytic promoters of Saccharomyces cerevisiae (Sc), Saccharomyces kudriavzevii (Sk), and Saccharomyces eubayanus (Se) were PCR-amplified using primers with specific Bsal flanks. First a 'GFP dropout' plasmid was assembled from the following parts containing all unique overhangs for assembly: two connectors ConLS and ConR, URA3 marker, $5^{\prime}$ and $3^{\prime}$ URA3 flanks and the Amp-ColE1 containing the marker and origin of replication for Escherichia coli. This plasmid was used in a second round of Bsal Golden Gate assembly to replace the GFP fragment by the promoter of interest, mRuby2, and ENO2 terminator. The resulting plasmids were linearized by Notl restriction and integrated in the ura3 locus of $S$. cerevisiae strain IMX1076.

independent duplicate. Batch cultures were performed in SMG supplemented with $0.2 \mathrm{~g} \mathrm{~L}^{-1}$ antifoam Emulsion C (SigmaAldrich). The reactors were inoculated at a starting $\mathrm{OD}_{660}$ of 0.3 with cells resuspended in demineralized water, which were obtained from exponentially growing shake flask cultures incubated at the same temperature and with the same medium as was used in the bioreactors (SMG). Cultures were performed in 2 L bioreactors (Applikon, Schiedam, The Netherlands) containing a $1.4 \mathrm{~L}$ working volume. The cultures were constantly stirred at $800 \mathrm{rpm}$, sparged with $700 \mathrm{~mL} \mathrm{~min}^{-1}$ dried compressed air (Linde Gas Benelux, Schiedam, The Netherlands) and maintained at $30^{\circ} \mathrm{C}$ for $S$. cerevisiae and $25^{\circ} \mathrm{C}$ for S. kudriavzevii and S. eubayanus. The culture $\mathrm{pH}$ was kept at 5.0 during growth on glucose by automatic addition of $2 \mathrm{M} \mathrm{KOH}$.

Extracellular metabolites were determined by highperformance liquid chromatography (HPLC) analysis using a Aminex HPX-87H ion-exchange column operated at $60^{\circ} \mathrm{C}$ with $5 \mathrm{mM} \mathrm{H}_{2} \mathrm{SO}_{4}$ as the mobile phase at a flow rate of $0.6 \mathrm{~mL} \mathrm{~min}^{-1}$ (Agilent, Santa Clara). Samples were centrifuged for $3 \mathrm{~min}$ at $20.000 \mathrm{~g}$ and the supernatant was used for analysis.

Biomass dry weight was determined in analytical duplicate by filtration of $10 \mathrm{~mL}$ sample on filters (pore-size $0.45 \mu \mathrm{m}$, Whatman/GE Healthcare Life Sciences, Little Chalfont, United Kingdom) pre-dried in a microwave oven at $360 \mathrm{~W}$ for $20 \mathrm{~min}$, as previously described (Verduyn et al., 1992). Optical density at $660 \mathrm{~nm}\left(\mathrm{OD}_{660}\right)$ was determined in a Libra S11 spectrophotometer (Biocrom, Cambridge, United Kingdom).
The $\mathrm{CO}_{2}$ and $\mathrm{O}_{2}$ concentration in the gas outflow was analyzed by a Rosemount NGA 2000 analyser (Baar, Switzerland), after cooling of the gas by a condenser $\left(2^{\circ} \mathrm{C}\right)$ and drying using a PermaPure Dryer (model MD 110-8P-4; Inacom Instruments, Veenendaal, Netherlands). Sampling for transcriptome analysis was done during mid-exponential growth on glucose at a biomass concentration of approximately $1 \mathrm{~g} \mathrm{~L}^{-1}$. Sampling in liquid nitrogen and RNA extraction were performed as previously described (Piper et al., 2002).

\section{Promoter Activity Assay}

Promoter activity measurement of the $m R$ uby 2 reporter strain library was performed in 96-well plates. Precultures were grown in 12-well plates in $1.5 \mathrm{~mL}$ volume in a thermoshaker (Grant-bio PHMP-4, United Kingdom) with constant shaking (800 rpm) and temperature. Precultures were grown at the temperature of the subsequent plate assay $\left(30^{\circ} \mathrm{C}\right.$ or $\left.20^{\circ} \mathrm{C}\right)$. For the first preculture YPD medium was inoculated from glycerol stocks and grown overnight till saturation. From this culture $20 \mu \mathrm{L}$ were transferred to new 12 -well plates and the strains were grown under the conditions of interest till mid-exponential phase (corresponding to $\mathrm{OD}_{660}$ of 3 to 5). Afterward the culture was centrifuged at $3000 \mathrm{~g}$ for $5 \mathrm{~min}$, the supernatant was removed and cells were resuspended in fresh medium to an $\mathrm{OD}_{660}$ of 0.3 and transferred in volumes of $100 \mu \mathrm{L}$ to a 96-well plate (Corning ${ }^{\mathrm{TM}}$ polystyrene white/transparent bottom, Greiner Bio-One) using six replicate wells per strain. To prevent evaporation, all plates, 
including preculture plates, were covered with sterile polyester acrylate sealing tape (Thermo Scientific). To supply sufficient levels of oxygen throughout the cultures, small openings were created in each well with a needle. The plate assays were performed in a plate reader (TECAN infinite M200 Pro. Tecan, Männedorf, Switzerland) with constant temperature and shaking (orbital, $1 \mathrm{~mm}$ ). Every $20 \mathrm{~min}$ the optical density $\left(\mathrm{OD}_{660}\right)$ and the fluorescence using excitation and emission wavelengths $559 \mathrm{~nm} / 600 \mathrm{~nm}$ were measured. Cultures were monitored till saturation. A non-fluorescent CEN.PK113-7D strain was taken along every run to determine the background fluorescence, as well as two reference reporter strains expressing $m R u b y 2$ from the TEF1 and ACT1 promoters from S. cerevisiae. For every well, $\mathrm{OD}_{660}$ and fluorescence values from all time points during exponential growth were plotted against each other and the promoter activity was calculated as the slope of the linear regression between optical density and fluorescence.

\section{Flow Cytometry Analysis}

mRuby2 fluorescence intensity of individual cells from cultures grown in the TECAN plate reader was determined using flow cytometry. Mid-exponential cultures from the plate reader were diluted in Isoton II (Beckman Coulter, Brea, CA) and the fluorescence intensity was determined for 10000 cells per sample on a BD FACSAriaII (Franklin Lakes, NJ) equipped with an $561 \mathrm{~nm}$ excitation laser and 582/15 nm emission filter. Data were analyzed using FlowJo v10.2 (FlowJo LLC). As expected from strains in which the $m R u b y 2$ expression system is integrated in the genome the fluorescence signal was homogeneously distributed among the yeast population (Supplementary Figure S5).

\section{Whole Genome Sequencing}

To obtain genome sequences of high quality, the strain S. kudriavzevii CR85 was sequenced in-house both by Illumina Miseq sequencing (Illumina, San Diego, CA) and by Oxford Nanopore Technology MinION sequencing (Oxford Nanopore Technology, Oxford, United Kingdom). Genomic DNA was isolated using the Qiagen 100/G kit (Qiagen, Hilden, Germany) and the concentration was determined using Qubit ${ }^{\circledR}$ Fluorometer 2.0 (ThermoFisher Scientific). Illumina library preparation was done as described previously (Świat et al., 2017).

For Nanopore sequencing, $3 \mu \mathrm{g}$ of genomic DNA were diluted in a total volume of $46 \mathrm{uL}$ and then sheared with a g-TUBE (Covaris, Brighton, United Kingdom) to an average fragment size of 8-10 kb. The input DNA was then prepared for loading in a FLO-MIN106 flow cell with R9.4 chemistry and the 1D ligation sequencing kit (SQK-LSK108), following manufacturer's instructions with the exception of a size selection step with $0.4 \mathrm{x}$ (instead of $1 \mathrm{x}$ ) AMPure beads after the End-Repair/dA tailing module and the use of $80 \%$ (instead of 70\%) ethanol for washes. Raw files generated by MinKNOW were base called using Albacore (version 1.2.5; Oxford Nanopore Technology). Reads, in fastq format, with minimum length of 1000 bp were extracted, yielding 4.15 Gigabase sequence with an average read length of $4.3 \mathrm{~kb}$.

De novo assembly was performed using Canu (v1.4, settings: genomesize $=12 \mathrm{~m})($ Koren et al., 2017) producing an 11.87
Megabase genome into 20 contigs of which 13 contigs in chromosome length plus 1 mitochondrial DNA, while 3 chromosomes consisted of 2 contigs each. The contig pairs were manually joined (with 1000 N's between the contigs) into 3 chromosomes (chromosomes VII, XII, and XVI). Pilon (Walker et al., 2014) was then used to further correct assembly errors by aligning Illumina reads, using BWA (Li and Durbin, 2010) to the assembly using correction of only SNPs and short indels (-fix bases parameter). Gene annotations were performed using the MAKER2 annotation pipeline (version 2.31.9) (Holt and Yandell, 2011) using SNAP (version 2013-11-29) (Korf, 2004) and Augustus (version 3.2.3) (Stanke and Waack, 2003) as $a b$ initio gene predictors. S288C EST and protein sequences were obtained from SGD (Saccharomyces Genome Database ${ }^{1}$ ) and were aligned using BLASTX (BLAST version 2.2.28+) (Camacho et al., 2009). The translated protein sequence of the final gene model was aligned using BLASTP to S288C protein Swiss-Prot database $^{2}$. For CEN.PK113-7D and S. eubayanus CBS 12357 existing sequencing data was used (Baker et al., 2015; Salazar et al., 2017). The sequencing data are available at NCBI under bioproject accession number PRJNA480800.

\section{RNA Sequencing and Data Analysis}

Library preparation and RNA sequencing were performed by Novogene Bioinformatics Technology Co., Ltd. (Yuen Long, Hong Kong). Sequencing was done with Illumina paired end 150 bp sequencing read system (PE150) using a 250 300 bp insert strand specific library which was prepared by Novogene. For the library preparation, as described by Novogene, mRNA enrichment was done using oligo(dT) beads. After random fragmentation of the mRNA, cDNA was synthetized from the mRNA using random hexamers primers. Afterward, second strand synthesis was done by addition of a custom second strand synthesis buffer (Illumina), dNTPs, RNase H and DNA polymerase I. Finally, after terminal repair, A ligation and adaptor ligation, the double stranded cDNA library was finalized by size selection and PCR enrichment.

The sequencing data for the three strains, S. cerevisiae CEN.PK122, S. kudriavzevii CR85 and S. eubayanus CBS 12357 obtained by Novogene had an average read depth of 21, 24 , and 24 million reads per sample, respectively. For each sample, reads were aligned to the relevant reference genome using a two-pass STAR procedure (Dobin et al., 2013). In the first pass, we assembled a splice junction database which was used to inform the second round of alignments. As paralogs in the glycolytic pathways were highly similar, we used stricter criteria for aligning and counting reads to facilitate delineation of paralogs. Introns were allowed to be between 15 and $4000 \mathrm{bp}$, and soft clipping was disabled to prevent low quality reads from being spuriously aligned. Ambiguously mapped reads were removed. Expression was quantified per transcript using htseq-count in strict intersection mode (Anders et al., 2015). As we wished to compare gene expression across genomes, where orthologs may have different gene lengths,

\footnotetext{
${ }^{1}$ http://www.yeastgenome.org

${ }^{2}$ http://www.ebi.ac.uk/swissprot/
} 
data were normalized for gene length. Therefore the average FPKM expression counts for each gene in each species were calculated (Trapnell et al., 2010). The genomes from S. cerevisiae CEN.PK113-7D, S. kudriavzevii CR85 and S. eubayanus CBS 12357 were used as reference NCBI BioProject accession numbers PRJNA52955, PRJNA480800, and PRJNA264003 respectively ${ }^{3}$. Data are available at Gene Expression Omnibus with accession number GSE117404. CEN.PK113-7D transcriptome data is available on Gene Expression Omnibus database under accession number GSE63884.

\section{Comparison of DNA Sequences}

Sequences from annotated glycolytic ORF and promoters of S. cerevisiae CEN.PK113-7D, S. kudriavzevii CR85 and S. eubayanus CBS 12357 were used for alignments with Clone Manager 9 Professional Edition, NCBI BioProject accession numbers PRJNA52955, PRJNA480800, and PRJNA264003 respectively. For the TPI1 sequence alignment the sequences with the following accession numbers were used: CU928179 (Z. rouxii), HE605205 (C. parapsilosis), CP028453 (Y. lipolytica), AJ390491 (C. albicans), XM_002551264 (C. tropicalis), AJ012317 (K. lactis), FR839630 (P. pastoris) AWRI1499 (D. bruxellensis), XM_ 018355487 (O. parapolymorpha), CR380954 (C. glabrata), CP002711 (A. gossypii), AP014602 (K. marxianus), XM_ 001642913 (K. polysporus), CP000501 (S. stipitis), XM_002616396 (C. lusitaniae), and CP028714 (E. coli).

Alignment of these sequences was performed using multiple sequence alignment in Clustal Omega (Goujon et al., 2010; Sievers et al., 2011) and the phylogenetic trees were obtained with JalView (version 2.10.4b1) using average distance and percentage identity (Waterhouse et al., 2009).

\section{Statistics}

Statistical analysis was performed using the software IBM SPSS statistics 23 (SPSS inc. Chicago). For transcriptome data, fluorescence data and batch culture data analysis of variance (ANOVA) with Dunnett post-hoc test was performed to test if the results for $S$. kudriavzevii and S. eubayanus were statistically different from S. cerevisiae.

\section{RESULTS}

\section{Genetic Makeup of the Glycolytic and Fermentative Pathways in S. cerevisiae and Its Close Relatives S. kudriavzevii and S. eubayanus}

The genetic makeup of pathways involved in central carbon metabolism in S. cerevisiae has already been well characterized, and more particularly for glycolysis and alcoholic fermentation. The ten reactions of the glycolytic pathway and the two reactions of ethanolic fermentation in S. cerevisiae are catalyzed by a set of 26 enzymes encoded by 26 genes (Figure 2). High quality sequences are already available for $S$. cerevisiae and $S$. eubayanus

${ }^{3}$ https://www.ncbi.nlm.nih.gov/bioproject/
(Baker et al., 2015; Salazar et al., 2017). To explore these pathways in S. kudriavzevii the strain S. kudriavzevii CR85 was sequenced using both Illumina and Oxford Nanopore technologies (see Materials and Methods section and Supplementary Table S5). $S$. cerevisiae's high genetic redundancy and the locations of the genes were fully mirrored in S. kudriavzevii and S. eubayanus genomes (Figure 1). The only exception was the absence of PDC6 in S. kudriavzevii. While a ScPDC6 ortholog with $81 \%$ identity was identified in S. eubayanus, no ortholog could be found in S. kudriavzevii. For all other glycolytic genes from S. cerevisiae, genes with $80-97 \%$ homology of the coding regions were found in S. kudriavzevii and S. eubayanus (Figure 2). Overall, genes from $S$. eubayanus were slightly more distant from their S. cerevisiae orthologs than genes from $S$. kudriavzevii, which is in line with earlier reports (Dujon, 2010; Shen et al., 2016; Figure 2).

In addition to the coding regions, the promoter regions were compared. Since the exact length of most promoter regions is not clearly defined, the 800 bp upstream of the coding regions were considered as promoters. Promoter sequences were substantially less conserved than the coding sequences, ranging from 43 to $78 \%$ identity when comparing S. kudriavzevii and S. eubayanus to $S$. cerevisiae promoters (Figure 2). Remarkably, some regions covering up to $45 \mathrm{bp}$ were strictly conserved among the three species, whereas other parts of the promoter sequences hardly shared homology (see example of PGK1p on Supplementary Figure S1). As promoter regions are poorly defined, promoters shorter than $800 \mathrm{bp}$ might be fully functional. Alignment with shorter regions might therefore increase the degree of homology between promoters. Alignments using 500 bp upstream the coding region only slightly increased the alignment percentages (up to 7\%), mostly as a consequence of the enrichment for conserved transcription factor binding sites located between 100 and 500 bp upstream of the ORF (Harbison et al., 2004). Notably, orthologs with a relatively high or low degree of conservation between $S$. cerevisiae and $S$. kudriavzevii also displayed a similar pattern when comparing S. eubayanus to $S$. cerevisiae. For example, the SkGPM2 and SeGPM2 promoters both have a relatively low homology (49 and 53\%) to the ScGPM2 promoter, whereas the $S k P F K 1$ and $S e P F K 1$ promoter have both a high degree (76 and 74\%) of similarity to ScPFK1. Interestingly, the genes and promoters displaying a relatively low degree of homology between $S$. cerevisiae and its relatives, are homologs considered as minor in S. cerevisiae (for example GPM2 and PYK2) (Figure 2). Blast searches did not identify additional glycolytic orthologs present in S. eubayanus or S. kudriavzevii but absent in S. cerevisiae.

The activity of a promoter strongly depends on the presence of regulatory sequences as the TATA box and other specific transcription factor binding sites. In $S$. cerevisiae, the most important glycolytic transcription factor is Gcrl, which has been experimentally shown to bind to most glycolytic promoters and to activate the expression of the corresponding genes as summarized before (Chambers et al., 1995). Gcr1 binding sites are only active when located next to DNA consensus sequences bound by Rap1 (Drazinic et al., 1996), a more pleiotropic transcription factor involved in the transcriptional regulation of a wide variety of genes including many glycolytic genes (Chambers 


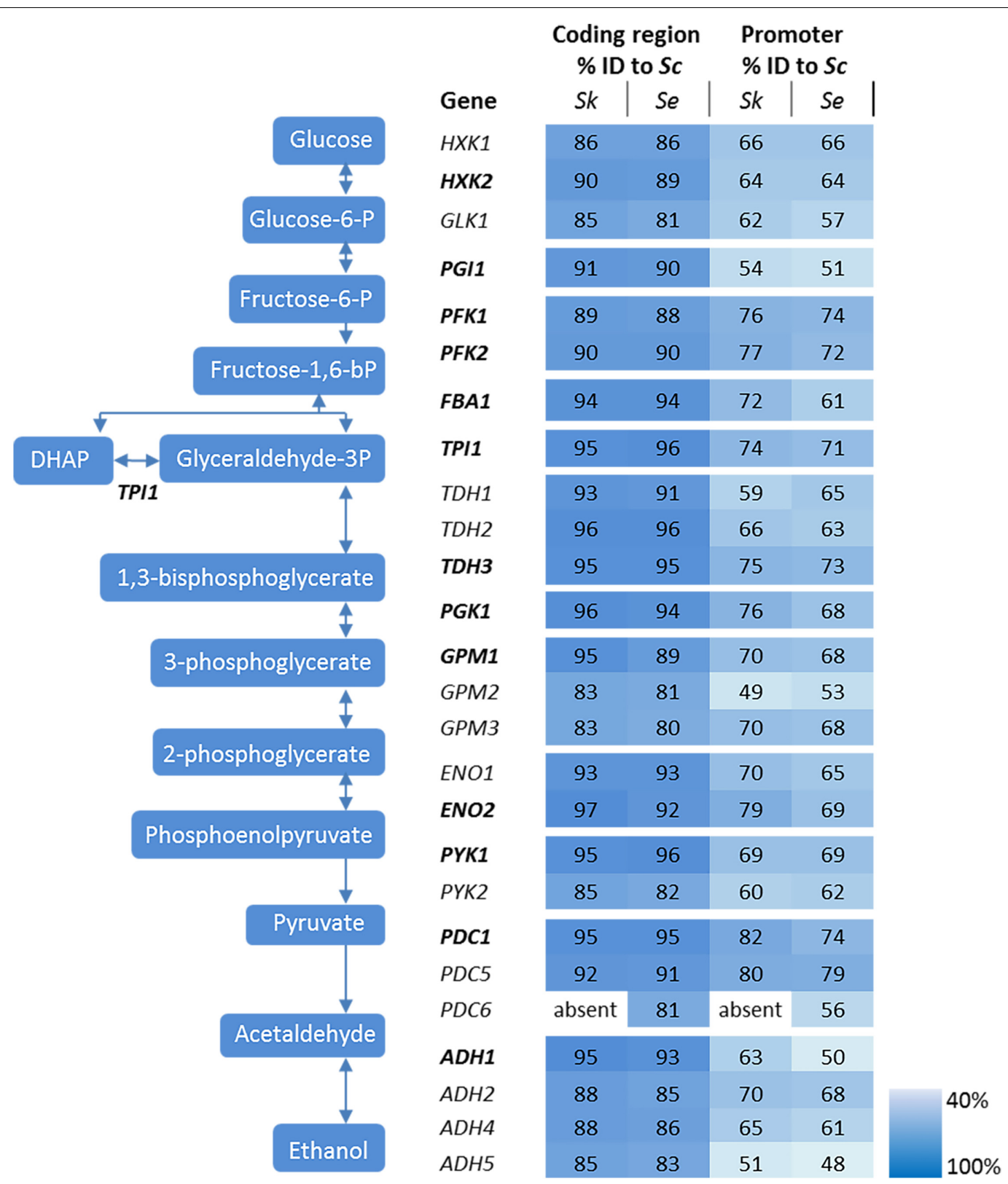

FIGURE 2 | Genes and reactions involved in glycolysis and alcoholic fermentation in S. cerevisiae and sequence comparison between the promoters and coding regions of $S$. cerevisiae $(S c)$, S. kudriavzevii (Sk), and S. eubayanus (Se). The major paralogs in S. cerevisiae are represented in bold. The coding regions and promoter regions (800 bp) of $S$. kudriavzevii and $S$. eubayanus were aligned to the corresponding $S$. cerevisiae sequences and the percentage identity is indicated. PDC6 was absent in S. kudriavzevii. The color scale indicates the degree of sequence identity between S. cerevisiae and its relatives.

et al., 1995). Another multifunctional transcription factor is Abf1 which binds to several glycolytic promoters (Chambers et al., 1995). With a single exception, all binding sites for Rap1, Gcr1, and Abf1 which were experimentally proven to be active in $S$. cerevisiae, were conserved in S. kudriavzevii and S. eubayanus promoter regions (Figure 3). The exception was the SeADH1 promoter in which the Rap1 and Gcrl site which are conserved between S. cerevisiae and S. kudriavzevii could not be identified. Together with the presence and high protein similarity of the SeRap1 (82\%), SkRap1 (86\%), SeGcr1 (85\%), and SkGcr1
(85\%), proteins with S. cerevisiae, these results suggested that the regulation of the glycolytic genes might be similar in the three species.

\section{Expression of the Glycolytic Genes During Aerobic Batch Cultivation}

To evaluate the similarity in glycolytic and fermentative gene expression, the transcriptome of S. cerevisiae, S. kudriavzevii, and S. eubayanus was compared. S. kudriavzevii and S. eubayanus are 


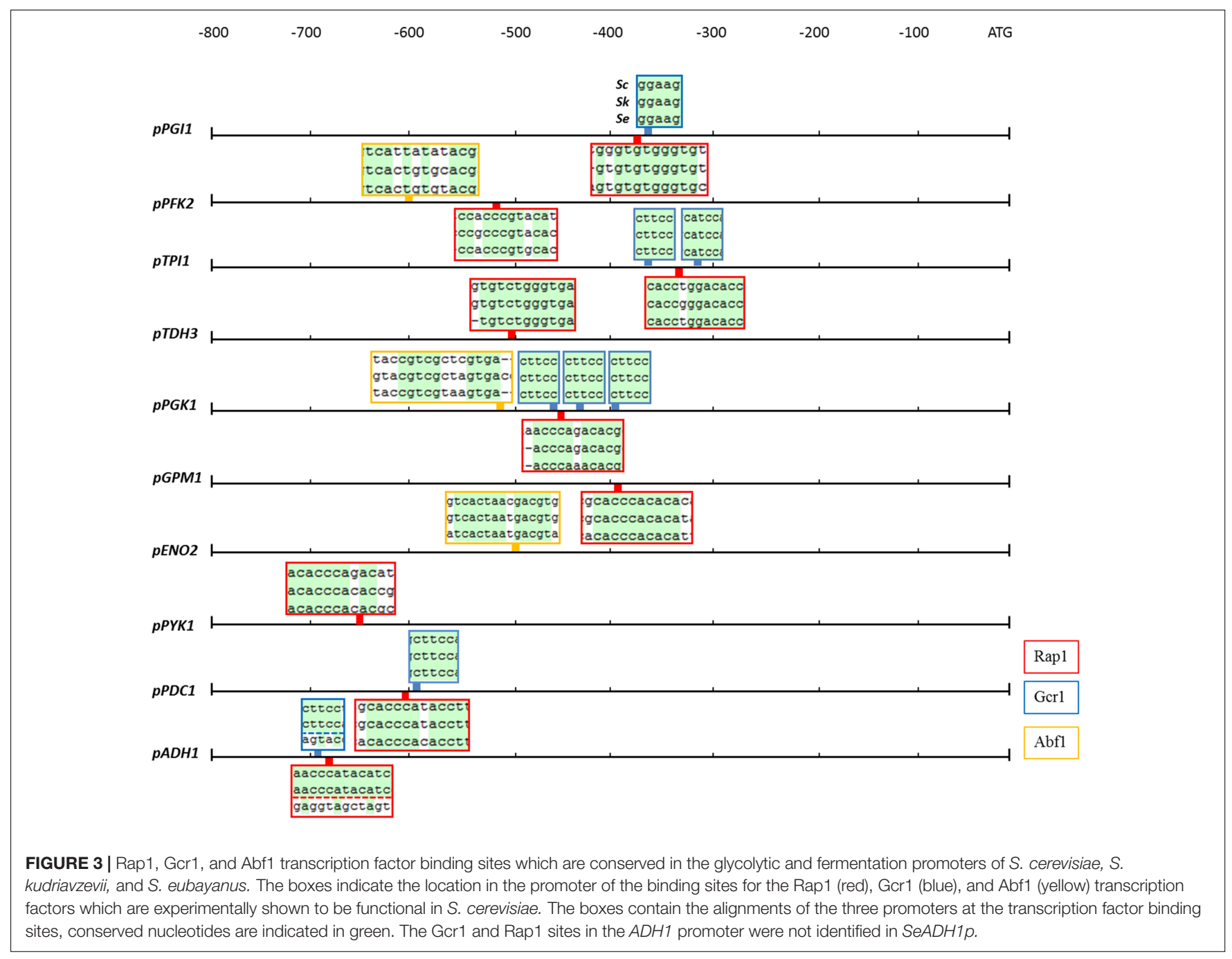

both wild isolates and both diploid (Lopes et al., 2010; Libkind et al., 2011). While many studies report the transcriptome of haploid S. cerevisiae strains, transcriptome data for diploid S. cerevisiae are scarce (Galitski et al., 1999; Li et al., 2010). To obtain comparable transcriptome datasets for the three species, the diploid CEN.PK122 strain was used. The three diploid strains were grown in aerobic batch cultures in bioreactor using minimal chemically defined medium with glucose as sole carbon source. To ensure optimal growth conditions S. cerevisiae was cultivated at $30^{\circ} \mathrm{C}$, while its coldtolerant relatives that have lower temperature optima were cultivated at $25^{\circ} \mathrm{C}$ (Arroyo-López et al., 2009; Hebly et al., 2015). Under these conditions the maximum specific growth rate of $S$. cerevisiae, S. kudriavzevii, and $S$. eubayanus was $0.38 \mathrm{~h}^{-1}, 0.25 \mathrm{~h}^{-1}$, and $0.33 \mathrm{~h}^{-1}$ respectively (Figure 4). Ethanol yields were similar for the three strains, but the biomass yield of S. kudriavzevii was significantly lower than that of its two relatives (Figure 4), which might reflect the higher relative cost of maintenance requirements at slow growth rates (Pirt, 1982). For S. eubayanus we observed a lower glycerol yield as compared to its relatives, which was previously not observed under anaerobic conditions (Hebly et al., 2015).

Transcriptome analysis of S. cerevisiae, S. kudriavzevii, and $S$. eubayanus during mid-exponential growth phase revealed a remarkable similarity between the three species (Figure 5), despite differences in culture temperature. Furthermore, the major or minor classification of paralogous genes was fully conserved between the three species (Figure 5). From the genes considered as major paralogs the SePFK1, SeFBA1, SkTDH3, SeTDH3, SkGPM1, SeENO2, and SeADH1 genes displayed significantly lower expression levels as compared to $S$. cerevisiae, although only for $S e T D H 3$ and $S e A D H 1$ the difference with $S$. cerevisiae was larger than 2 -fold (8 and 3-fold, respectively). For the minor paralogs slightly more variability was observed. Interestingly SeHXK1 expression was 13-fold higher than its $S$. cerevisiae ortholog. All three $T D H$ genes displayed a significantly lower expression in S. kudriavzevii and S. eubayanus as compared to $S$. cerevisiae. Likewise, for ENO1 a lower expression was observed for SeENO1 and even lower for SkENO1 as compared to $S c E N O 1$. Finally, compared to $S$. cerevisiae a ca. 3-fold higher expression was observed for SkPDC5 and SeADH4. 

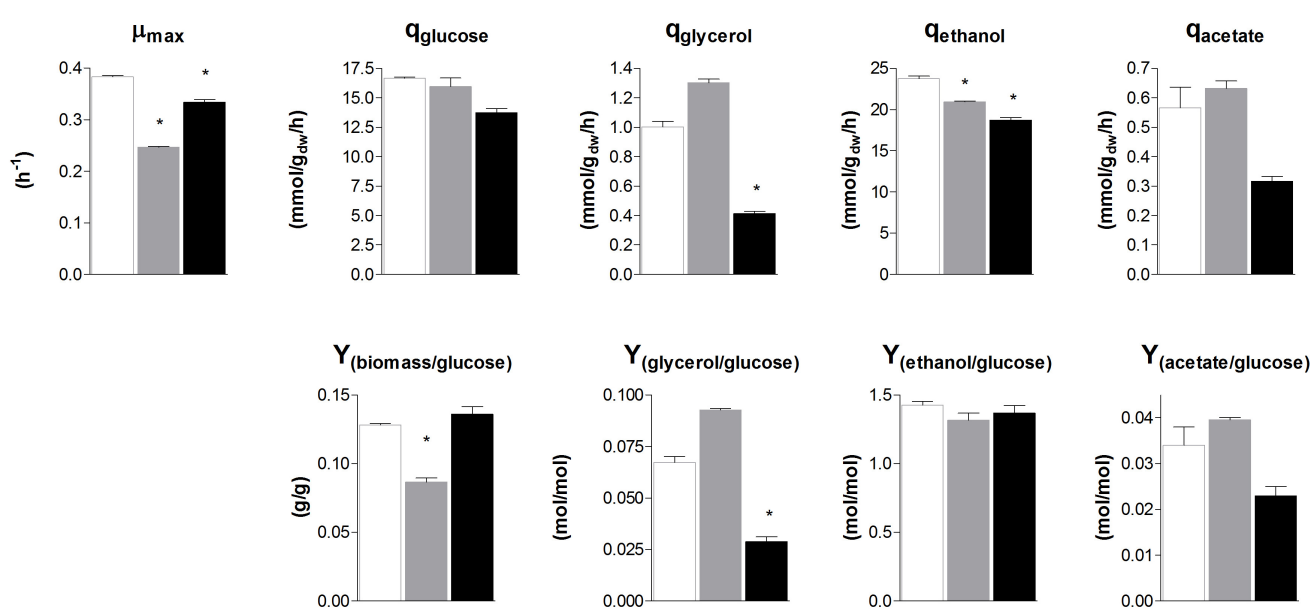

FIGURE 4 | Biomass specific rates and yields of S. cerevisiae, S. kudriavzevii, and S. eubayanus batch cultivations in bioreactor. The strains were grown aerobically in synthetic medium supplemented with $20 \mathrm{~g} \mathrm{~L}^{-1}$ glucose. S. cerevisiae CEN.PK122 (white) was grown at $30^{\circ} \mathrm{C}$, and S. kudriavzevii CR85 (gray) and S. eubayanus CBS 12357 (black) at $25^{\circ} \mathrm{C}$. Asterisks indicate significant difference from S. cerevisiae (One-Way ANOVA, Dunnett post hoc test, $P<0.01$ ).

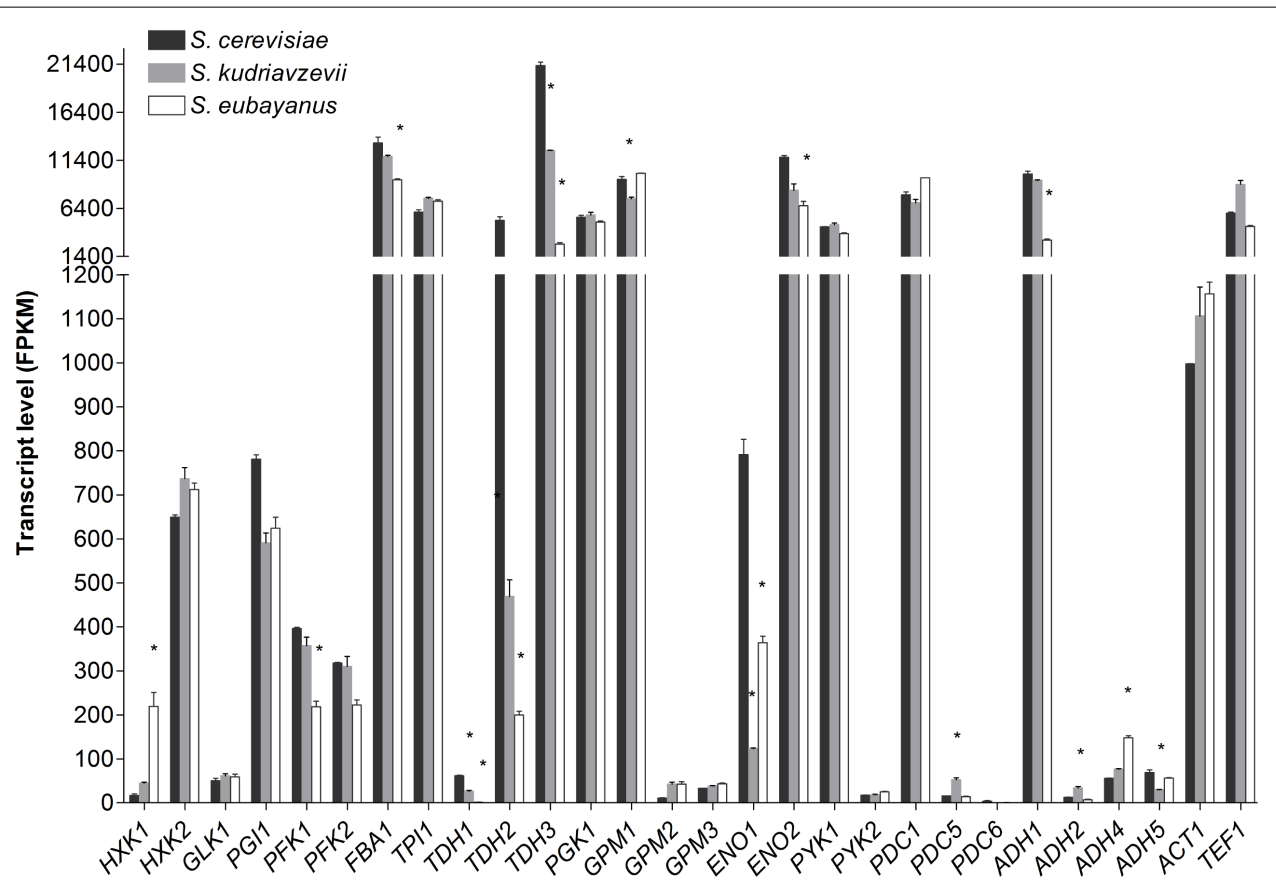

FIGURE 5 | Transcript levels of the diploid strains S. cerevisiae (black), S. kudriavzevii (gray), and S. eubayanus (white) from two biological replicates during mid-exponential growth in aerobic batch fermentations on glucose. Asterisks indicate significant difference from S. cerevisiae per gene (One-Way ANOVA, Dunnett post hoc test, $P<0.01)$.

\section{Optimization of Microtiter Plate Assays to Monitor Promoter Strength via Fluorescent Reporters}

To explore the transferability of promoters within the Saccharomyces genus, the promoters of the major glycolytic and fermentative genes (indicated in bold in Figure 2) of S. kudriavzevii and S. eubayanus were functionally characterized in S. cerevisiae. A library of fluorescent reporter strains in which $m R$ uby 2 expression was driven by heterologous promoters and, for comparison, by $S$. cerevisiae promoters, was constructed. To avoid bias due to gene copy number, the constructs were integrated in S. cerevisiae genome, at the URA3 locus. The strains were cultured in 96-well plates, sealed with a transparent foil to prevent evaporation. Simultaneous monitoring of optical density and fluorescence revealed a premature saturation of the fluorescence signal as compared to biomass formation (Supplementary Figures S2A,B). Fluorescent proteins have a 
strict requirement for molecular oxygen for the synthesis of their chromophores (Tsien, 1998). The poor oxygenation of the cultures in sealed plates combined with the competition for oxygen between cellular respiration, anabolic reactions and mRuby 2 maturation could explain the early saturation of the fluorescence signal. Unfortunately, this effect is rarely reported in literature and could be easily overlooked when fluorescence is measured at only one or few time points. Plate readers are widely used as method to characterize promoters with fluorescence reporters (Davis et al., 2010; Zeevi et al., 2011; Keren et al., 2013; Lee et al., 2015) however, information provided in Materials and Methods Sections are often scarce or incomplete, which makes reproduction of data by other groups difficult. To increase oxygen transfer while preventing evaporation, a small aperture was created in each well by puncturing the seal with a needle. The presence of an aperture had a strong impact on the fluorescence intensity of the cultures, enabling to monitor the cultures for a prolonged period of time (Supplementary Figure S2B). Also during growth with ethanol as sole carbon source, for which oxygen requirement is substantially increased, no premature saturation of fluorescence was observed (Supplementary Figures S2C,D). The location of the aperture in the well did not affect the fluorescence intensity (data not shown). To further evaluate the reliability of the fluorescence signal measured by the plate reader as well as the cell-to-cell heterogeneity of the fluorescence signal, measurements were also performed by flow cytometry. Comparing these data with the plate reader data revealed a very strong correlation of the fluorescence measured with these two techniques $\left(R^{2}=0,96\right.$, Supplementary Figure S3).

\section{Transferability and Context-Dependency of Glycolytic and Fermentative Promoters Within the Saccharomyces Genus}

The strain library grown in SMG at $30^{\circ} \mathrm{C}$ not only revealed that the S. kudriavzevii and $S$. eubayanus promoters could drive gene expression in $S$. cerevisiae, but also that their strength was remarkably similar to the strength of their S. cerevisiae orthologs (Figure 6). Additionally, two reporter strains expressing $m R u b y 2$ from the constitutive $S$. cerevisiae TEF1 and ACT1 promoters were constructed and cultivated on all plates experiments. The activity of these two promoters was remarkably reproducible between independent culture replicates (Supplementary Figure S4).

While, due to high data reproducibility, expression driven by S. kudriavzevii or S. eubayanus promoters was in most cases considered statistically different from the expression led by their $S$. cerevisiae orthologs (student $t$-test, $P<0.01$ ), differences in expression larger than 1.5-fold were rarely observed. Expression of ENO2p and PDC1p of S. kudriavzevii and S. eubayanus was lower than for their $S$. cerevisiae counterparts, while $S k G P M 1 p$, SeGPM1p, and SePYK1p led to clearly higher expression levels than their S. cerevisiae homologs (Figure 6). These differences were not reflected in the transcript data (Figure 5). Conversely, the differential expression of PFK1 and TDH3 revealed by the
RNAseq analysis was also found in the promoter transplantation study at $\mathrm{SMG} 30^{\circ} \mathrm{C}$. Overall similarities and differences between the three species in transcript levels were mirrored by promoter activity.

To test the condition dependency of promoter activity, strains were tested under several culture conditions. YPD was used as rich medium, and ethanol was used as gluconeogenic carbon source (SMEtOH). Since S. kudriavzevii and S. eubayanus have a lower optimum growth temperature and hexokinase from S. kudriavzevii has been proposed to have a lower temperature optimum as compared to S. cerevisiae (Gonçalves et al., 2011), the strains were also grown in SMG at $20^{\circ} \mathrm{C}$. When grown in YPD and SMG at $20^{\circ} \mathrm{C}$, all strains showed highly similar promoter activities as compared to cultures in SMG at $30^{\circ} \mathrm{C}$ even though the growth rates were different $\left(\mathrm{SMG} 30^{\circ} \mathrm{C}: 0.34 \mathrm{~h}^{-1}, \mathrm{SMG} 20^{\circ} \mathrm{C}\right.$ : $0.15 \mathrm{~h}^{-1}$, YPD $\left.30^{\circ} \mathrm{C}: 0.36 \mathrm{~h}^{-1}\right)$. However, during growth on ethanol $\left(0.13 \mathrm{~h}^{-1}\right)$ promoter activity of the three species dropped tremendously as compared to glucose-grown cultures, in stark contrast with the fluorescence of the reference strains (TEF1p and $A C T 1 \mathrm{p}$ ) that remained remarkably constant for all cultivation conditions. Nevertheless, also on SMEtOH S. kudriavzevii and S. eubayanus promoters showed expression levels very similar to their S. cerevisiae orthologs.

\section{DISCUSSION}

In this study we showed that the genetic makeup of the glycolytic and fermentative pathways is highly conserved among S. cerevisiae, S. kudriavzevii, and S. eubayanus. For 11 out of 12 reactions, the exact same number of paralogs was found in the three species, reflecting that species divergence took place after whole genome and post-whole genome duplications. The only exception was the absence of the minor paralog PDC6 in the S. kudriavzevii CR85 genome. In agreement with this observation, the presence of a pseudogene in S. kudriavzevii strains IFO1802 and ZP591 consisting of only about 15\% of the full PDC6 gene length has been reported (Scannell et al., 2011). At the transcript level a strong conservation was also observed, suggesting that the classification between major and minor paralogs, confirmed in $S$. cerevisiae by mutant studies, could be extended to S. kudriavzevii and S. eubayanus. The slightly lower degree of conservation of minor paralogs (e.g., GPM2, PYK2, ENO1, TDH1, TDH2, PDC6, ADH2, ADH4, ADH5) is in line with the previously reported accelerated evolution of the PYK2 and $A D H 5$ as compared to their PYK1 and $A D H 1$ paralogs (Kellis et al., 2004).

The glycolytic pathway is known to be highly conserved compared to most other pathways (Fothergill-Gilmore and Michels, 1993; Webster, 2003). Recently it was shown that glycolytic coding regions from $E$. coli could replace the corresponding yeast genes (Kachroo et al., 2017). For promoter regions the conservation is in general lower as compared to coding regions, but a stronger conservation was found for the glycolytic promoters in the Saccharomyces genus than for other promoter regions (Kuang et al., 2017). Combined with the remarkable conservation of binding sites 
A

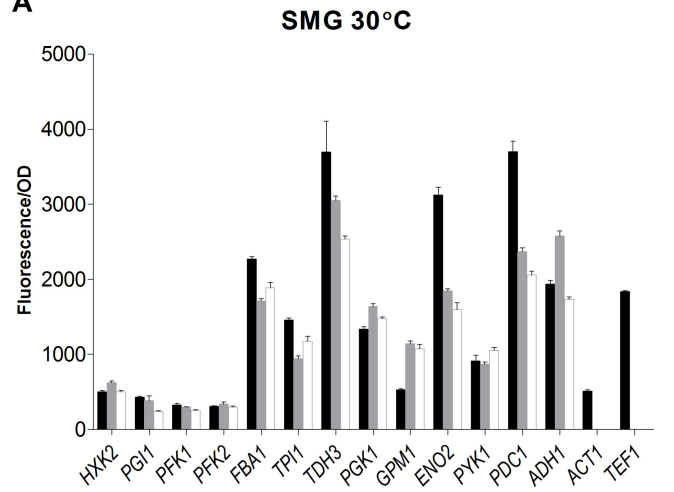

C

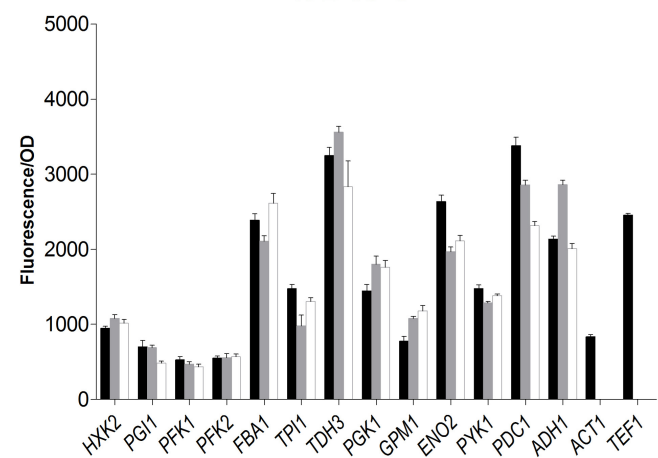

B

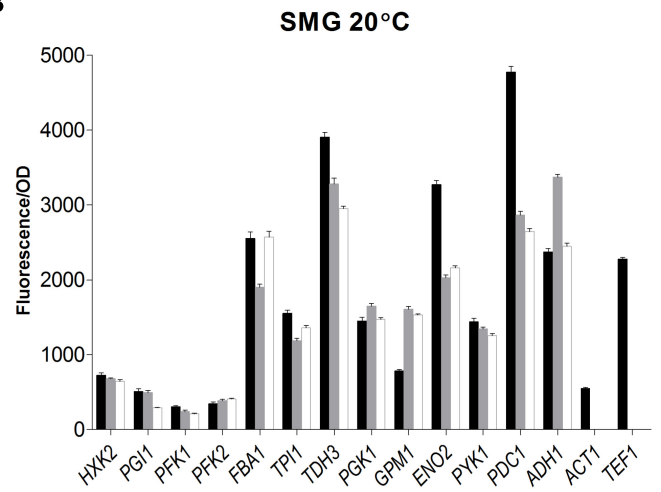

D

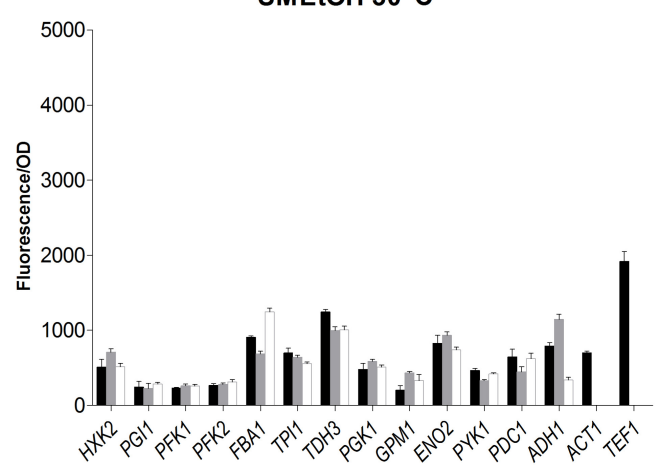

FIGURE 6 | Promoter activity of the major glycolytic promoters from S. cerevisiae (black), S. kudriavzevii (gray), and S. eubayanus (white) expressing mRuby2 in S. cerevisiae. During exponential growth in SMG (A), SMG $20^{\circ} \mathrm{C}$ (B), YPD (C), and SMEtOH (D) fluorescence and optical density were measured every 20 min and promoter activity was calculated as the slope of the linear regression between optical density and fluorescence. Two reference strains expressing $m R$ Ruby2 from the SCTEF1 and SCACT1 promoters were taken along in every plate. Error bars represent the standard deviation of the mean of six biological replicates.

for major transcriptional regulators (i.e., Rap1, Gcr1, and Abf1), these observations suggested a very similar transcriptional regulation of glycolytic and fermentative genes across the three species. Accordingly, transcriptome data showed a remarkable conservation in expression for the majority of glycolytic and fermentative genes in their native context. It is noteworthy that transcript levels of glycolytic and fermentative genes of these three diploid species were highly similar to the transcript levels of the haploid S. cerevisiae CEN.PK113-7D cultivated in the same condition as the $S$. cerevisiae diploid (Solis-Escalante et al., 2015). The similarity in gene expression and the conservation of the main transcription factor binding sites in the three species suggested the possibility to introduce the promoters in $S$. cerevisiae, expecting similar regulation.

Until now a limited number of examples of heterologous glycolytic promoters driving gene expression in S. cerevisiae is available. Recently, it was shown that $S$. kudriavzevii glycolytic and fermentative promoters could drive gene expression in S. cerevisiae (Kuijpers et al., 2016). More recently, it was shown that the ADH2 promoter of several Saccharomyces species could drive gene expression in S. cerevisiae (Harvey et al., 2018). Further, the glycolytic genes PFK1, PFK2 and PYK1 of the more distantly related yeast Hanseniaspora uvarum, expressed from their native promoters were shown to complement their S. cerevisiae orthologs (Langenberg et al., 2017).

To explore the conservation of glycolytic genes in a broader context, the sequence of the TPI1 gene was compared across a set of 18 species within the Saccharomycotina subphylum (Dujon, 2010). Within this subphylum, the coding region of TPI1 was highly conserved (ranging from 64,7 to $96,3 \%$ identity to $S$. cerevisiae), while the promoters generally displayed a much weaker similarity (ranging from 28,5\%-71,1\% identity to $S$. cerevisiae) (Figure 7). These observations are in line with studies reporting the loss of the gene encoding the Gcrl transcription factor and the gain of new function by Rap 1 in the CTG clade yeast Candida albicans (Askew et al., 2009; Lavoie et al., 2010; Weirauch and Hughes, 2010). Indeed using the MEME suite motif discovery tool (Bailey et al., 2009) gave only hits for Rap1 and Gcr1 motifs in the Saccharomyces genus.

The present study shows the ability of all the major glycolytic promoters of $S$. kudriavzevii and S. eubayanus to drive gene expression in $S$. cerevisiae with similar strength and conditiondependency. Since many hybrids occur between $S$. cerevisiae $x S$. eubayanus and $S$. cerevisiae $x$ S. kudriavzevii, it is 


\section{A}

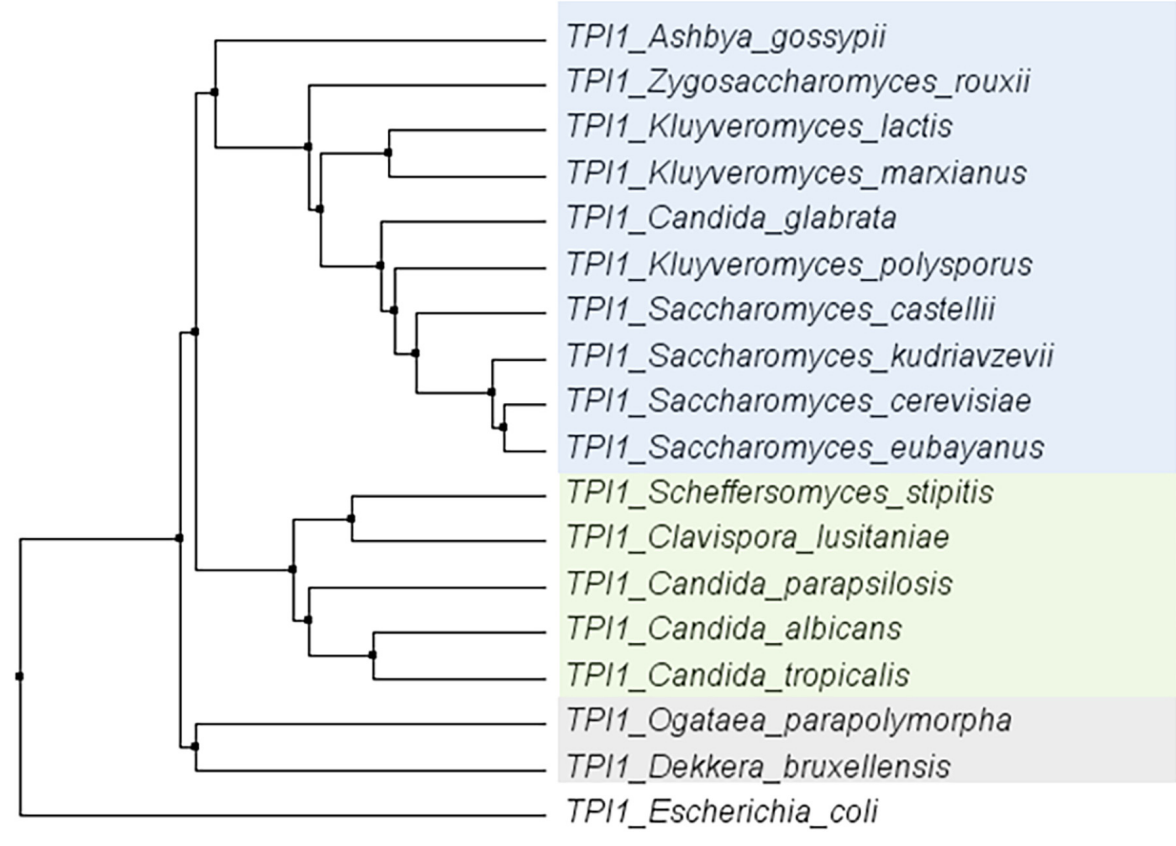

B

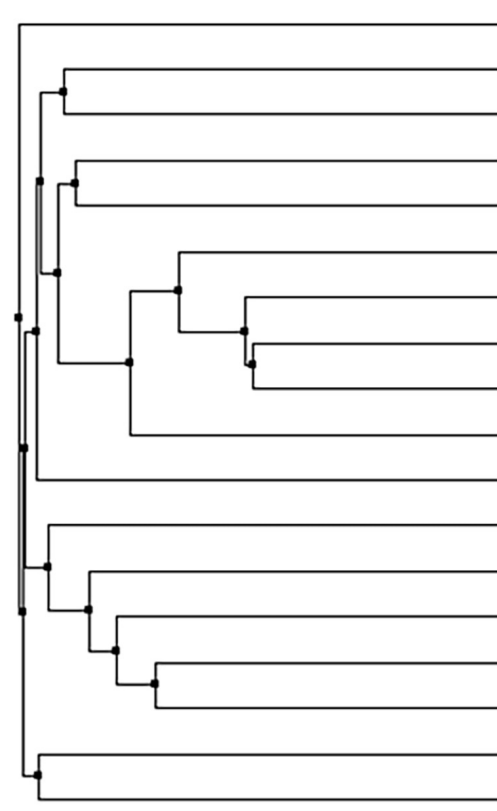

pTPI1_Candida_glabrata

pTPI1_Ashbya_gossypii

pTPI1_Ogataea_parapolymorpha

pTPI1_Kluyveromyces_polysporus

pTPI1_Candida_parapsilosis

pTPI1_Zygosaccharomyces_rouxii

pTPI1_Saccharomyces_eubayanus

pTPI1_Saccharomyces_cerevisiae

pTPI1_Saccharomyces_kudriavzevii

pTPI1_Saccharomyces_castellii

pTPI1_Scheffersomyces_stipitis

pTPI1_Dekkera_bruxellensis

pTPI1_Clavispora_lusitaniae

pTPI1_Kluyveromyces_lactis

pTPI1_Candida_albicans

pTPI1_Candida_tropicalis

pTPI1_Escherichia_coli

pTPI1_Kluyveromyces_marxianus

FIGURE 7 | Phylogenetic trees of the alignments of the TPl1 genes (ORF) (A) and promoters (800 bp) (B) for a set of yeast species from the Saccharomycotina phylum. The coding regions are strongly conserved, whereas there is hardly any conservation among promoter regions. E. coli was used as an outgroup. Color indicates groups as defined in Dujon (2010).

not surprising that promoters are functional in S. cerevisiae. However, the similarity we found in promoter activities for most promoters transplanted to $S$. cerevisiae under different conditions is remarkable and indicates a strong conservation of the glycolytic regulatory mechanisms for $S$. cerevisiae, $S$. kudriavzevii, and S. eubayanus (Supplementary Figure S6). In general, these data do not correlate very well with the transcript data (Supplementary Figure S7). This can most likely be explained by the relatively low dynamic measurement range of the plate reader compared to RNAseq, differences in cultivation conditions, length of promoters, choice of site for genomic integration (Bai Flagfeldt et al., 2009) or differences in regulatory sequences in the promoters. During growth on ethanol a strong decrease in promoter activity was observed. 
This is in agreement with the previously reported drop in enzymatic activity of glycolysis during growth on ethanol (Peter Smits et al., 2000; Van Hoek et al., 2000).

S. cerevisiae's proficiency in assembling and functionally expressing large (heterologous) pathways has propelled this yeast as preferred host for the production of complex molecules such as isoprenoids or opioids (Paddon et al., 2013; Galanie et al., 2015). However, the successful expression of these pathways depends on the availability of suitable promoters. While $S$. cerevisiae has one of the most furbished molecular toolbox, the number of constitutive and well characterized promoters remains limited. Since $S$. cerevisiae's extremely efficient homologous recombination renders strains with repeated usage of promoter sequences genetically unstable (Manivasakam et al., 1995), this shortage of promoters presents a hurdle for extensive strain construction programs. While a lot of effort is invested in the design of synthetic promoters and transcription amplifiers (Redden and Alper, 2015; Rantasalo et al., 2016; Machens et al., 2017; Naseri et al., 2017), using slightly distant but functional orthologous promoters presents an attractive alternative (Naesby et al., 2009; Harvey et al., 2018). Usage of especially the S. eubayanus promoters, which are slightly more distant from $S$. cerevisiae than the $S$. kudriavzevii promoters, would reduce the length of the sequences being $100 \%$ identical to the native $S$. cerevisiae promoters. The minimum length which was found to be needed for efficient homologous recombination in S. cerevisiae was $30 \mathrm{bp}$ with an optimal efficiency at a length of $60 \mathrm{bp}$ or more (Manivasakam et al., 1995; Hua et al., 1997). In the S. eubayanus promoters, with one exception for the PFK2 promoter, the longest sequence being identical to $S$. cerevisiae was found to be $34 \mathrm{bp}$. Usage of the S. eubayanus promoters would therefore substantially decrease the risk of instability and undesired recombination events during strain construction programs.

\section{CONCLUSION}

This study brings new insight in the genetic makeup and expression of glycolytic and fermentative genes in S. eubayanus and S. kudriavzevii. It also expands the molecular toolbox for $S$. cerevisiae, but also for its two relatives, with a set of strong,

\section{REFERENCES}

Anders, S., Pyl, P. T., and Huber, W. (2015). HTSeq-a Python framework to work with high-throughput sequencing data. Bioinformatics 31, 166-169. doi: 10.1093/bioinformatics/btu638

Arroyo-López, F. N., Orlić, S., Querol, A., and Barrio, E. (2009). Effects of temperature, $\mathrm{pH}$ and sugar concentration on the growth parameters of Saccharomyces cerevisiae, S. kudriavzevii and their interspecific hybrid. Int. J. Food Microbiol. 131, 120-127. doi: 10.1016/j.ijfoodmicro.2009. 01.035

Arroyo-López, F. N., Pérez-Torrado, R., Querol, A., and Barrio, E. (2010). Modulation of the glycerol and ethanol syntheses in the yeast Saccharomyces kudriavzevii differs from that exhibited by Saccharomyces cerevisiae and their hybrid. Food Microbiol. 27, 628-637. doi: 10.1016/j.fm.2010.02.001 constitutive promoters. Furthermore, combining Illumina and Oxford Nanopore technologies, the present study offers a high quality sequence for $S$. kudriavzevii CR85, available from NCBI (PRJNA480800). Finally, the full set of transcript levels for the three diploid strains grown in tightly controlled conditions is available via GEO (See Materials and Methods section) and can be mined to compare species-specific regulation of gene expression beyond the glycolytic and fermentative pathways.

\section{AUTHOR CONTRIBUTIONS}

FB, J-MD, and PD-L designed the research. FB and SD performed the experiments. MvdB and TG performed the transcriptome analysis. MvdB performed the sequence analysis. FB, SD, J-MD, and PD-L prepared the manuscript. All authors read and approved the final manuscript.

\section{FUNDING}

This project was funded by the AdLibYeast European Research Council (ERC) consolidator 648141 grant awarded to PD-L.

\section{ACKNOWLEDGMENTS}

We thank Rik Brouwer for his contribution to strain construction, Mark Bisschops for advice and help with fermentations and data analysis, Marijke Luttik for technical support, and advice for the TECAN plate reader and flow cytometry analysis and Pilar de la Torre for performing the whole genome sequencing. Furthermore, we thank Jack Pronk for his advice and Raúl Ortiz Merino for constructive comments on the manuscript. We thank Eladio Barrio Esparducer for kindly providing S. kudriavzevii CR85.

\section{SUPPLEMENTARY MATERIAL}

The Supplementary Material for this article can be found online at: https://www.frontiersin.org/articles/10.3389/fgene. 2018.00504/full\#supplementary-material

Arvanitidis, A., and Heinisch, J. J. (1994). Studies on the function of yeast phosphofructokinase subunits by in vitro mutagenesis. J. Biol. Chem. 269, 8911-8918.

Askew, C., Sellam, A., Epp, E., Hogues, H., Mullick, A., Nantel, A., et al. (2009). Transcriptional regulation of carbohydrate metabolism in the human pathogen Candida albicans. PLoS Path. 5:e1000612. doi: 10.1371/journal.ppat.1000612

Bai Flagfeldt, D., Siewers, V., Huang, L., and Nielsen, J. (2009). Characterization of chromosomal integration sites for heterologous gene expression in Saccharomyces cerevisiae. Yeast 26, 545-551. doi: 10.1002/yea.1705

Bailey, T. L., Boden, M., Buske, F. A., Frith, M., Grant, C. E., Clementi, L., et al. (2009). MEME SUITE: tools for motif discovery and searching. Nucleic Acids Res. 37, W202-W208. doi: 10.1093/nar/gkp335

Baker, E., Wang, B., Bellora, N., Peris, D., Hulfachor, A. B., Koshalek, J. A., et al. (2015). The genome sequence of Saccharomyces eubayanus and 
the domestication of lager-brewing yeasts. Mol. Biol. Evol. 32, 2818-2831. doi: $10.1093 / \mathrm{molbev} / \mathrm{msv} 168$

Barnett, J. A. (2003). A history of research on yeasts 5: the fermentation pathway. Yeast 20, 509-543. doi: 10.1002/yea.986

Barnett, J. A., and Entian, K. D. (2005). A history of research on yeasts 9: regulation of sugar metabolism. Yeast 22, 835-894. doi: 10.1002/yea.1249

Belloch, C., Orlic, S., Barrio, E., and Querol, A. (2008). Fermentative stress adaptation of hybrids within the Saccharomyces sensu stricto complex. Int. J. Food Microbiol. 122, 188-195. doi: 10.1016/j.ijfoodmicro.2007.11.083

Bertani, G. (1951). Studies on lysogenis I.: the mode of phage liberation by lysogenic Escherichia coli. J. Bacteriol. 62, 293-300.

Bertani, G. (2004). Lysogeny at mid-twentieth century: P1. P2, and other experimental systems. J. Bacteriol. 186, 595-600. doi: 10.1128/JB.186.3.595-600. 2004

Boer, V. M., De Winde, J. H., Pronk, J. T., and Piper, M. D. (2003). The genomewide transcriptional responses of Saccharomyces cerevisiae grown on glucose in aerobic chemostat cultures limited for carbon, nitrogen, phosphorus, or sulfur. J. Biol. Chem. 278, 3265-3274. doi: 10.1074/jbc.M209759200

Bond, U. (2009). The genomes of lager yeasts. Adv. Appl. Microbiol. 69, 159-182. doi: 10.1016/S0065-2164(09)69006-7

Camacho, C., Coulouris, G., Avagyan, V., Ma, N., Papadopoulos, J., Bealer, K., et al. (2009). BLAST + : architecture and applications. BMC Bioinformatics 10:421. doi: 10.1186/1471-2105-10-421

Carroll, K. M., Simpson, D. M., Eyers, C. E., Knight, C. G., Brownridge, P., Dunn, W. B., et al. (2011). Absolute quantification of the glycolytic pathway in yeast: deployment of a complete QconCAT approach. Mol. Cell. Proteomics 10:M111.007633. doi: 10.1074/mcp.M111.007633

Chambers, A., Packham, E. A., and Graham, I. R. (1995). Control of glycolytic gene expression in the budding yeast (Saccharomyces cerevisiae). Curr. Genet. 29, 1-9. doi: 10.1007/BF00313187

Ciriacy, M. (1979). Isolation and characterization of further cis-and trans-acting regulatory elements involved in the synthesis of glucose-repressible alcohol dehydrogenase (ADHII) in Saccharomyces cerevisiae. Mol. Gen. Genet. 176, 427-431. doi: 10.1007/BF00333107

Conant, G. C., and Wolfe, K. H. (2007). Increased glycolytic flux as an outcome of whole - genome duplication in yeast. Mol. Syst. Biol. 3:129. doi: 10.1038/ msb4100170

Conant, G. C., and Wolfe, K. H. (2008). Turning a hobby into a job: how duplicated genes find new functions. Nat. Rev. Genet. 9, 938-950. doi: 10.1038/nrg2482

Davis, J. H., Rubin, A. J., and Sauer, R. T. (2010). Design, construction and characterization of a set of insulated bacterial promoters. Nucleic Acids Res. 39, 1131-1141. doi: 10.1093/nar/gkq810

De Deken, R. (1966). The Crabtree effect: a regulatory system in yeast. Microbiology 44, 149-156. doi: 10.1099/00221287-44-2-149

Dobin, A., Davis, C. A., Schlesinger, F., Drenkow, J., Zaleski, C., Jha, S., et al. (2013). STAR: ultrafast universal RNA-seq aligner. Bioinformatics 29, 15-21. doi: 10.1093/bioinformatics/bts635

Drazinic, C. M., Smerage, J. B., López, M. C., and Baker, H. V. (1996). Activation mechanism of the multifunctional transcription factor repressor-activator protein 1 (Rap1p). Mol. Cell. Biol. 16, 3187-3196. doi: 10.1128/MCB.16.6.3187

Dujon, B. (2010). Yeast evolutionary genomics. Nat. Rev. Genet. 11, 512-524. doi: $10.1038 / \mathrm{nrg} 2811$

Entian, K.-D., and Kötter, P. (2007). 25 Yeast genetic strain and plasmid collections. Methods Microbiol. 36, 629-666. doi: 10.1016/S0580-9517(06)36025-4

Fauchon, M., Lagniel, G., Aude, J.-C., Lombardia, L., Soularue, P., Petat, C., et al. (2002). Sulfur sparing in the yeast proteome in response to sulfur demand. Mol. Cell 9, 713-723. doi: 10.1016/S1097-2765(02)00500-2

Fothergill-Gilmore, L. A., and Michels, P. A. (1993). Evolution of glycolysis. Prog. Biophys. Mol. Biol. 59, 105-235. doi: 10.1016/0079-6107(93)90001-Z

Fraenkel, D. G. (2003). The top genes: on the distance from transcript to function in yeast glycolysis. Curr. Opin. Microbiol. 6, 198-201. doi: 10.1016/S1369-5274(03) 00023-7

Galanie, S., Thodey, K., Trenchard, I. J., Interrante, M. F., and Smolke, C. D. (2015). Complete biosynthesis of opioids in yeast. Science 349, 1095-1100. doi: $10.1126 /$ science.aac 9373

Galitski, T., Saldanha, A. J., Styles, C. A., Lander, E. S., and Fink, G. R. (1999). Ploidy regulation of gene expression. Science 285, 251-254. doi: 10.1126/science.285. 5425.251
Gietz, R. D., and Woods, R. A. (2002). Transformation of yeast by lithium acetate/single-stranded carrier DNA/polyethylene glycol method. Methods Enzymol. 350, 87-96. doi: 10.1016/S0076-6879(02)50957-5

Gonçalves, P., Valério, E., Correia, C., de Almeida, J. M., and Sampaio, J. P. (2011). Evidence for divergent evolution of growth temperature preference in sympatric Saccharomyces species. PLoS One 6:e20739. doi: 10.1371/journal. pone. 0020739

González, S. S., Barrio, E., Gafner, J., and Querol, A. (2006). Natural hybrids from Saccharomyces cerevisiae, Saccharomyces bayanus and Saccharomyces kudriavzevii in wine fermentations. FEMS Yeast Res. 6, 1221-1234. doi: 10. 1111/j.1567-1364.2006.00126.x

González, S. S., Barrio, E., and Querol, A. (2008). Molecular characterization of new natural hybrids of Saccharomyces cerevisiae and S. kudriavzevii in brewing. Appl. Environ. Microbiol. 74, 2314-2320. doi: 10.1128/AEM.01867-07

Goujon, M., McWilliam, H., Li, W., Valentin, F., Squizzato, S., Paern, J., et al. (2010). A new bioinformatics analysis tools framework at EMBL-EBI. Nucleic Acids Res. 38, W695-W699. doi: 10.1093/nar/gkq313

Harbison, C. T., Gordon, D. B., Lee, T. I., Rinaldi, N. J., Macisaac, K. D., Danford, T. W., et al. (2004). Transcriptional regulatory code of a eukaryotic genome. Nature 431, 99-104. doi: 10.1038/nature02800

Harvey, C. J., Tang, M., Schlecht, U., Horecka, J., Fischer, C. R., Lin, H.-C., et al. (2018). HEx: a heterologous expression platform for the discovery of fungal natural products. Sci. Adv. 4:eaar5459. doi: 10.1126/sciadv.aar5459

Hebly, M., Brickwedde, A., Bolat, I., Driessen, M. R., de Hulster, E. A., van den Broek, M., et al. (2015). S. cerevisiae $\times$ S. eubayanus interspecific hybrid, the best of both worlds and beyond. FEMS Yeast Res. 15:fov005. doi: 10.1093/femsyr/ fov005

Heinisch, J., Vogelsang, K., and Hollenberg, C. P. (1991). Transcriptional control of yeast phosphofructokinase gene expression. FEBS Lett. 289, 77-82. doi: 10. 1016/0014-5793(91)80912-M

Hittinger, C. T. (2013). Saccharomyces diversity and evolution: a budding model genus. Trends Genet. 29, 309-317. doi: 10.1016/j.tig.2013.01.002

Holt, C., and Yandell, M. (2011). MAKER2: an annotation pipeline and genomedatabase management tool for second-generation genome projects. BMC Bioinformatics 12:491. doi: 10.1186/1471-2105-12-491

Hua, S.-B., Qiu, M., Chan, E., Zhu, L., and Luo, Y. (1997). Minimum length of sequence homology required for in vivo cloning by homologous recombination in yeast. Plasmid 38, 91-96. doi: 10.1006/plas.1997.1305

Kachroo, A. H., Laurent, J. M., Akhmetov, A., Szilagyi-Jones, M., McWhite, C. D., Zhao, A., et al. (2017). Systematic bacterialization of yeast genes identifies a near-universally swappable pathway. Elife. 6:e25093. doi: 10.7554/eLife.25093

Kellis, M., Birren, B. W., and Lander, E. S. (2004). Proof and evolutionary analysis of ancient genome duplication in the yeast Saccharomyces cerevisiae. Nature 428, 617-624. doi: 10.1038/nature02424

Keren, L., Zackay, O., Lotan-Pompan, M., Barenholz, U., Dekel, E., Sasson, V., et al. (2013). Promoters maintain their relative activity levels under different growth conditions. Mol. Syst. Biol. 9:701. doi: 10.1038/msb.2013.59

Knijnenburg, T. A., Daran, J.-M. G., van den Broek, M. A., Daran-Lapujade, P. A., de Winde, J. H., Pronk, J. T., et al. (2009). Combinatorial effects of environmental parameters on transcriptional regulation in Saccharomyces cerevisiae: a quantitative analysis of a compendium of chemostat-based transcriptome data. BMC Genomics 10:53. doi: 10.1186/1471-2164-10-53

Koren, S., Walenz, B. P., Berlin, K., Miller, J. R., Bergman, N. H., and Phillippy, A. M. (2017). Canu: scalable and accurate long-read assembly via adaptive k-mer weighting and repeat separation. Genome Res. 27, 722-736. doi: 10.1101/ gr.215087.116

Korf, I. (2004). Gene finding in novel genomes. BMC Bioinformatics 5:59. doi: 10.1186/1471-2105-5-59

Kuang, Z., Pinglay, S., Ji, H., and Boeke, J. D. (2017). Msn2/4 regulate expression of glycolytic enzymes and control transition from quiescence to growth. Elife 6:e29938. doi: 10.7554/eLife.29938

Kuepfer, L., Sauer, U., and Blank, L. M. (2005). Metabolic functions of duplicate genes in Saccharomyces cerevisiae. Genome Res. 15, 1421-1430. doi: 10.1101/gr. 3992505

Kuijpers, N. G., Solis-Escalante, D., Luttik, M. A., Bisschops, M. M., Boonekamp, F. J., van den Broek, M., et al. (2016). Pathway swapping: toward modular engineering of essential cellular processes. Proc. Natl. Acad. Sci. U.S.A. 113, 15060-15065. doi: 10.1073/pnas.1606701113 
Langenberg, A.-K., Bink, F. J., Wolff, L., Walter, S., von Wallbrunn, C., Grossmann, M., et al. (2017). Glycolytic functions are conserved in the genome of the wine yeast Hanseniaspora uvarum and pyruvate kinase limits its capacity for alcoholic fermentation. Appl. Environ. Microbiol. 83:e01580-17. doi: 10. 1128/AEM.01580-17

Lavoie, H., Hogues, H., Mallick, J., Sellam, A., Nantel, A., and Whiteway, M. (2010). Evolutionary tinkering with conserved components of a transcriptional regulatory network. PLoS Biol. 8:e1000329. doi: 10.1371/journal.pbio.10 00329

Lee, M. E., DeLoache, W. C., Cervantes, B., and Dueber, J. E. (2015). A highly characterized yeast toolkit for modular, multipart assembly. ACS Synth. Biol. 4, 975-986. doi: 10.1021/sb500366v

Li, B.-Z., Cheng, J.-S., Ding, M.-Z., and Yuan, Y.-J. (2010). Transcriptome analysis of differential responses of diploid and haploid yeast to ethanol stress. J. Biotechnol. 148, 194-203. doi: 10.1016/j.jbiotec.2010.06.013

Li, H., and Durbin, R. (2010). Fast and accurate long-read alignment with BurrowsWheeler transform. Bioinformatics 26, 589-595. doi: 10.1093/bioinformatics/ btp698

Libkind, D., Hittinger, C. T., Valério, E., Gonçalves, C., Dover, J., Johnston, M., et al. (2011). Microbe domestication and the identification of the wild genetic stock of lager-brewing yeast. Proc. Natl. Acad. Sci. U.S.A. 108, 14539-14544. doi: 10.1073/pnas.1105430108

Lopes, C. A., Barrio, E., and Querol, A. (2010). Natural hybrids of S. cerevisiae $x$ S. kudriavzevii share alleles with European wild populations of Saccharomyces kudriavzevii. FEMS Yeast Res. 10, 412-421. doi: 10.1111/j.1567-1364.2010. 00614.x

Machens, F., Balazadeh, S., Mueller-Roeber, B., and Messerschmidt, K. (2017). Synthetic promoters and transcription factors for heterologous protein expression in Saccharomyces cerevisiae. Front. Bioeng. Biotechnol. 5:63. doi: 10.3389/fbioe.2017.00063

Manivasakam, P., Weber, S. C., McElver, J., and Schiestl, R. H. (1995). Microhomology mediated PCR targeting in Saccharomyces cerevisiae. Nucleic Acids Res. 23, 2799-2800. doi: 10.1093/nar/23.14.2799

Masneuf-Pomarède, I., Bely, M., Marullo, P., Lonvaud-Funel, A., and Dubourdieu, D. (2010). Reassessment of phenotypic traits for Saccharomyces bayanus var. uvarum wine yeast strains. Int. J. Food Microbiol. 139, 79-86. doi: 10.1016/j.ijfoodmicro.2010.01.038

Merico, A., Sulo, P., Piškur, J., and Compagno, C. (2007). Fermentative lifestyle in yeasts belonging to the Saccharomyces complex. FEBS J. 274, 976-989. doi: $10.1111 / \mathrm{j} .1742-4658.2007 .05645 . \mathrm{x}$

Naesby, M., Nielsen, S. V., Nielsen, C. A., Green, T., Tange, T. $\varnothing$, Simón, E., et al. (2009). Yeast artificial chromosomes employed for random assembly of biosynthetic pathways and production of diverse compounds in Saccharomyces cerevisiae. Microb. Cell Fact. 8:45. doi: 10.1186/1475-2859-8-45

Naseeb, S., James, S. A., Alsammar, H., Michaels, C. J., Gini, B., Nueno-Palop, C., et al. (2017). Saccharomyces jurei sp. nov., Isolation and genetic identification of a novel yeast species from Quercus robur. Int. J. Syst. Evol. Microbiol. 67, 2046-2052. doi: 10.1099/ijsem.0.002013

Naseri, G., Balazadeh, S., Machens, F., Kamranfar, I., Messerschmidt, K., and Mueller-Roeber, B. (2017). Plant-derived transcription factors for orthologous regulation of gene expression in the yeast Saccharomyces cerevisiae. ACS Synth. Biol. 6, 1742-1756. doi: 10.1021/acssynbio.7b00094

Nguyen, H. V., and Boekhout, T. (2017). Characterization of Saccharomyces uvarum (Beijerinck, 1898) and related hybrids: assessment of molecular markers that predict the parent and hybrid genomes and a proposal to name yeast hybrids. FEMS Yeast Res. 17:2. doi: 10.1093/femsyr/fox014

Nijkamp, J. F., van den Broek, M., Datema, E., de Kok, S., Bosman, L., Luttik, M. A., et al. (2012). De novo sequencing, assembly and analysis of the genome of the laboratory strain Saccharomyces cerevisiae CEN. PK113-7D, a model for modern industrial biotechnology. Microb. Cell Fact. 11:36. doi: 10.1186/14752859-11-36

Ohno, S. (1970). Evolution by Gene Duplication. New York, NY: Springer. doi: 10.1007/978-3-642-86659-3

Paddon, C. J., Westfall, P. J., Pitera, D. J., Benjamin, K., Fisher, K., McPhee, D., et al. (2013). High-level semi-synthetic production of the potent antimalarial artemisinin. Nature 496, 528-532. doi: 10.1038/nature12051

Peng, B., Williams, T. C., Henry, M., Nielsen, L. K., and Vickers, C. E. (2015). Controlling heterologous gene expression in yeast cell factories on different carbon substrates and across the diauxic shift: a comparison of yeast promoter activities. Microb. Cell Fact. 14:91. doi: 10.1186/s12934-015-0278-5

Peris, D., Lopes, C. A., Belloch, C., Querol, A., and Barrio, E. (2012). Comparative genomics among Saccharomyces cerevisiae $\times$ Saccharomyces kudriavzevii natural hybrid strains isolated from wine and beer reveals different origins. BMC Genomics 13:407. doi: 10.1186/1471-2164-13-407

Peter Smits, H., Hauf, J., Müller, S., Hobley, T. J., Zimmermann, F. K., HahnHägerdal, B., et al. (2000). Simultaneous overexpression of enzymes of the lower part of glycolysis can enhance the fermentative capacity of Saccharomyces cerevisiae. Yeast 16, 1325-1334. doi: 10.1002/1097-0061(200010)16:14<1325:: AID-YEA627>3.0.CO;2-E

Piper, M. D., Daran-Lapujade, P., Bro, C., Regenberg, B., Knudsen, S., Nielsen, J., et al. (2002). Reproducibility of oligonucleotide microarray transcriptome analyses an interlaboratory comparison using chemostat cultures of Saccharomyces cerevisiae. J. Biol. Chem. 277, 37001-37008. doi: 10.1074/jbc. M204490200

Pirt, S. (1982). Maintenance energy: a general model for energy-limited and energysufficient growth. Arch. Microbiol. 133, 300-302. doi: 10.1007/BF00521294

Rantasalo, A., Czeizler, E., Virtanen, R., Rousu, J., Lähdesmäki, H., Penttilä, M., et al. (2016). Synthetic transcription amplifier system for orthogonal control of gene expression in Saccharomyces cerevisiae. PLoS One 11:e0148320. doi: 10.1371/journal.pone.0148320

Redden, H., and Alper, H. S. (2015). The development and characterization of synthetic minimal yeast promoters. Nat. Common. 6:7810. doi: 10.1038/ ncomms 8810

Replansky, T., Koufopanou, V., Greig, D., and Bell, G. (2008). Saccharomyces sensu stricto as a model system for evolution and ecology. Trends Ecol. Evol. 23, 494-501. doi: 10.1016/j.tree.2008.05.005

Salazar, A. N., Gorter de Vries, A. R., van den Broek, M., Wijsman, M., de la Torre Cortés, P., Brickwedde, A., et al. (2017). Nanopore sequencing enables nearcomplete de novo assembly of Saccharomyces cerevisiae reference strain CEN. PK113-7D. FEMS Yeast Res. 17:fox074. doi: 10.1093/femsyr/fox074

Salvadó, Z., Arroyo-López, F., Guillamón, J., Salazar, G., Querol, A., and Barrio, E. (2011). Temperature adaptation markedly determines evolution within the genus Saccharomyces. Appl. Environ. Microbiol. 77, 2292-2302. doi: 10.1128/ AEM.01861-10

Scannell, D. R., Zill, O. A., Rokas, A., Payen, C., Dunham, M. J., Eisen, M. B., et al. (2011). The awesome power of yeast evolutionary genetics: new genome sequences and strain resources for the Saccharomyces sensu stricto genus. G3 1, 11-25. doi: 10.1534/g3.111.000273

Shen, X.-X., Zhou, X., Kominek, J., Kurtzman, C. P., Hittinger, C. T., and Rokas, A. (2016). Reconstructing the backbone of the Saccharomycotina yeast phylogeny using genome-scale data. G3 6, 3927-3939. doi: 10.1534/g3.116.034744

Sicard, D., and Legras, J.-L. (2011). Bread, beer and wine: yeast domestication in the Saccharomyces sensu stricto complex. C. R. Biol. 334, 229-236. doi: 10.1016/ j.crvi.2010.12.016

Sievers, F., Wilm, A., Dineen, D., Gibson, T. J., Karplus, K., Li, W., et al. (2011). Fast, scalable generation of high-quality protein multiple sequence alignments using Clustal Omega. Mol. Syst. Biol. 7:539. doi: 10.1038/msb.2011.75

Solis-Escalante, D., Kuijpers, N. G., Barrajon-Simancas, N., van den Broek, M., Pronk, J. T., Daran, J. M., et al. (2015). A minimal set of glycolytic genes reveals strong redundancies in Saccharomyces cerevisiae central metabolism. Eukaryot. Cell 14, 804-816. doi: 10.1128/EC.00064- 15

Stanke, M., and Waack, S. (2003). Gene prediction with a hidden Markov model and a new intron submodel. Bioinformatics 19, ii215-ii225. doi: 10.1093/ bioinformatics/btg1080

Świat, M. A., Dashko, S., den Ridder, M., Wijsman, M., van der Oost, J., Daran, J.-M., et al. (2017). FnCpf1: a novel and efficient genome editing tool for Saccharomyces cerevisiae. Nucleic Acids Res. 45, 12585-12598. doi: 10.1093/nar/ gkx1007

Trapnell, C., Williams, B. A., Pertea, G., Mortazavi, A., Kwan, G., Van Baren, M. J., et al. (2010). Transcript assembly and quantification by RNA-Seq reveals unannotated transcripts and isoform switching during cell differentiation. Nat. Biotechnol. 28, 511-515. doi: 10.1038/nbt.1621

Tsien, R. Y. (1998). The green fluorescent protein. Annu. Rev. Biochem. 67, 509-544. doi: 10.1146/annurev.biochem.67.1.509

Van Dijken, J., Bauer, J., Brambilla, L., Duboc, P., Francois, J., Gancedo, C., et al. (2000). An interlaboratory comparison of physiological and genetic properties 
of four Saccharomyces cerevisiae strains. Enzyme Microb. Technol. 26, 706-714. doi: 10.1016/S0141-0229(00)00162-9

Van Heerden, J. H., Bruggeman, F. J., and Teusink, B. (2015). Multi-tasking of biosynthetic and energetic functions of glycolysis explained by supply and demand logic. Bioessays 37, 34-45. doi: 10.1002/bies.201400108

Van Hoek, P., Van Dijken, J. P., and Pronk, J. T. (2000). Regulation of fermentative capacity and levels of glycolytic enzymes in chemostat cultures of Saccharomyces cerevisiae. Enzyme Microb. Technol. 26, 724-736. doi: 10.1016/ S0141-0229(00)00164-2

Verduyn, C., Postma, E., Scheffers, W. A., and Van Dijken, J. P. (1992). Effect of benzoic acid on metabolic fluxes in yeasts: a continuous-culture study on the regulation of respiration and alcoholic fermentation. Yeast 8, 501-517. doi: $10.1002 /$ yea. 320080703

Walker, B. J., Abeel, T., Shea, T., Priest, M., Abouelliel, A., Sakthikumar, S., et al. (2014). Pilon: an integrated tool for comprehensive microbial variant detection and genome assembly improvement. PLoS One 9:e112963. doi: 10.1371/journal. pone. 0112963

Waterhouse, A. M., Procter, J. B., Martin, D. M., Clamp, M., and Barton, G. J. (2009). Jalview Version 2-a multiple sequence alignment editor and analysis workbench. Bioinformatics 25, 1189-1191. doi: 10.1093/bioinformatics/btp033

Webster, K. A. (2003). Evolution of the coordinate regulation of glycolytic enzyme genes by hypoxia. J. Exp. Biol. 206, 2911-2922. doi: 10.1242/jeb.00516
Weirauch, M. T., and Hughes, T. R. (2010). Conserved expression without conserved regulatory sequence: the more things change, the more they stay the same. Trends Genet. 26, 66-74. doi: 10.1016/j.tig.2009.12.002

Wolfe, K. H., and Shields, D. C. (1997). Molecular evidence for an ancient duplication of the entire yeast genome. Nature 387, 708-713. doi: 10.1038/ 42711

Zeevi, D., Sharon, E., Lotan-Pompan, M., Lubling, Y., Shipony, Z., Raveh-Sadka, T., et al. (2011). Compensation for differences in gene copy number among yeast ribosomal proteins is encoded within their promoters. Genome Res. 21, 2114-2128. doi: 10.1101/gr.119669.110

Conflict of Interest Statement: The authors declare that the research was conducted in the absence of any commercial or financial relationships that could be construed as a potential conflict of interest.

Copyright (c) 2018 Boonekamp, Dashko, van den Broek, Gehrmann, Daran and Daran-Lapujade. This is an open-access article distributed under the terms of the Creative Commons Attribution License (CC BY). The use, distribution or reproduction in other forums is permitted, provided the original author(s) and the copyright owner(s) are credited and that the original publication in this journal is cited, in accordance with accepted academic practice. No use, distribution or reproduction is permitted which does not comply with these terms. 